Draft VERsion April 28, 2018

Preprint typeset using $\mathrm{AT}_{\mathrm{E} X} \mathrm{X}$ style emulateapj v. 01/23/15

\title{
PROBING THE INNERMOST REGIONS OF AGN JETS AND THEIR MAGNETIC FIELDS WITH RADIOASTRON. I. IMAGING BL LACERTAE AT 21 MICROARCSECOND RESOLUTION
}

\author{
José L. Gómez ${ }^{1}$, Andrei P. Lobanov ${ }^{2,3}$, Gabriele Bruni ${ }^{2}$, Yuri Y. Kovalev ${ }^{4,2}$, Alan P. Marscher ${ }^{5}$, Svetlana G. \\ Jorstad $^{5,6}$, Yosuke Mizuno ${ }^{7}$, Uwe Bach ${ }^{2}$, Kirill V. Sokolovsky ${ }^{4,8,9}$, James M. Anderson ${ }^{2,10}$, Pablo Galindo $^{1}$, \\ Nikolay S. KardasheV ${ }^{4}$, AND Mikhail M. LisaKov ${ }^{4}$ \\ Draft version April 28, 2018
}

\begin{abstract}
We present the first polarimetric space VLBI imaging observations at $22 \mathrm{GHz}$. BL Lacertae was observed in 2013 November 10 with the RadioAstron space VLBI mission, including a ground array of 15 radio telescopes. The instrumental polarization of the space radio telescope is found to be within $9 \%$, demonstrating the polarimetric imaging capabilities of RadioAstron at $22 \mathrm{GHz}$. Groundspace fringes were obtained up to a projected baseline distance of 7.9 Earth's diameters in length, allowing us to image the jet in BL Lacertae with a maximum angular resolution of $21 \mu$ as, the highest achieved to date. We find evidence for emission upstream of the radio core, which may correspond to a recollimation shock at about $40 \mu$ as from the jet apex, in a pattern that includes other recollimation shocks at approximately $100 \mu$ as and $250 \mu$ as from the jet apex. Polarized emission is detected in two components within the innermost 0.5 mas from the core, as well as in some knots 3 mas downstream. Faraday rotation analysis, obtained from combining RadioAstron $22 \mathrm{GHz}$ and ground-based $15 \mathrm{GHz}$ and $43 \mathrm{GHz}$ images, shows a gradient in rotation measure and Faraday corrected polarization vector as a function of position angle with respect to the core, suggesting that the jet in BL Lacertae is threaded by a helical magnetic field. The intrinsic de-boosted brightness temperature in the unresolved core exceeds $3 \times 10^{12} \mathrm{~K}$, suggesting at the very least departure from equipartition of energy between the magnetic field and radiating particles.

Subject headings: galaxies: active - galaxies: individual (BL Lac) - galaxies: jets - polarization radio continuum: galaxies
\end{abstract}

\section{INTRODUCTION}

Accretion of gas onto the supermassive black holes lurking at the center of active galactic nuclei (AGN) gives rise to powerful relativistic jets (e.g., Marscher et al. 2002). These are produced by dynamically important magnetic fields twisted by differential rotation of the black hole's accretion disk or ergosphere (Blandford \& Znajek 1977; Blandford \& Payne 1982; McKinney \& Blandford 2009 Zamaninasab et al. 2014). Observational signatures for the existence of such helical magnetic fields can be obtained by looking for Faraday rota-

\footnotetext{
${ }^{1}$ Instituto de Astrofísica de Andalucía-CSIC, Glorieta de la Astronomía s/n, 18008 Granada, Spain. jlgomez@iaa.csic.es

${ }^{2}$ Max-Planck-Institut für Radioastronomie, Auf dem Hügel 69, 53121 Bonn, Germany

${ }^{3}$ Institut für Experimentalphysik, Universität Hamburg, Luruper Chaussee 149, 22761 Hamburg, Germany

${ }_{4}$ Astro Space Center, Lebedev Physical Institute, Russian Academy of Sciences, Profsoyuznaya str. 84/32, Moscow 117997, Russia

${ }^{5}$ Institute for Astrophysical Research, Boston University, 725 Commonwealth Avenue, Boston, MA 02215

${ }^{6}$ Astronomical Institute, St. Petersburg State University, Universitetskij Pr. 28, Petrodvorets, 198504 St.Petersburg, Russia

${ }^{7}$ Institute for Theoretical Physics, Goethe University, 60438, Frankfurt am Main, Germany

8 Sternberg Astronomical Institute, Moscow State University, Universitetskii pr. 13, 119992 Moscow, Russia

${ }^{9}$ Institute of Astronomy, Astrophysics, Space Applications and Remote Sensing, National Observatory of Athens, Vas. Pavlou \& I. Metaxa, GR-15 236 Penteli, Greece

10 Helmholtz-Zentrum Potsdam, Deutsches GeoForschungsZentrum GFZ, Telegrafenberg, 14473 Potsdam, Germany
}

tion gradients, produced by the systematic change in the net line-of-sight magnetic field component across the jet width (Laing 1981; Asada et al. 2002).

Obtaining a better understanding of the jet formation, and of the role played by the magnetic field requires probing the innermost regions of AGN jets, but this is limited by the insufficient angular resolution provided by existing, ground-based, very long baseline interferometry (VLBI) arrays. However, space VLBI, in which one of the antennas is in Earth orbit, is capable of extending the baseline distances beyond the Earth's diameter, reaching unprecedentedly high angular resolutions in astronomical observations (e.g., Levy et al. 1986, Gómez \& Marscher 2000, Gabuzda \& Gómez 2001; Lobanov \& Zensus 2001).

On 2011 July 18, the RadioAstron space VLBI mission (Kardashev et al. 2013) began to operate, featuring a $10 \mathrm{~m}$ space radio telescope (SRT) on board the satellite Spektr-R. RadioAstron provides the first true fullpolarization capabilities for space VLBI observations on baselines longer than the Earth's diameter at 0.32, 1.6, and $22 \mathrm{GHz}$. The SRT operates also at $5 \mathrm{GHz}$, but an onboard hardware failure limits the recording mode at this frequency to left circular polarization only.

The current paradigm for AGN is that their radio emission is explained by synchrotron radiation from relativistic electrons that are Doppler boosted through bulk motion. In this model, the intrinsic brightness temperatures cannot exceed $10^{11}$ to $10^{12} \mathrm{~K}$ (Kellermann \& PaulinyToth 1969 Readhead 1994). Typical Doppler boosting is expected to be able to raise this temperature by a factor of $\sim 10$ (see also Hovatta et al. 2009, Lister et al. 2013). 
For direct interferometric measurements, increasing the interferometer baseline length is the only way to measure higher brightness temperatures (see e.g., Kovalev et al. 2005), and hence, to place stringent observational constraints on the physics of the most energetic relativistic outflows. The highest observing frequency, $22 \mathrm{GHz}$, and resolution of RadioAstron allow us to probe the most energetic regions located closer to the central engine (see, e.g., Lobanov 1998, Sokolovsky et al. 2011, Pushkarev et al. 2012) while scattering effects in the Galaxy are negligible (Pushkarev \& Kovalev 2015).

First polarimetric space VLBI imaging observations with RadioAstron were performed on 2013 March 9 during the early science program, targeting the highredshift quasar TXS $0642+449$ at a frequency of $1.6 \mathrm{GHz}$ (Lobanov et al. 2015). Instrumental polarization of the SRT was found to be smaller than $9 \%$ in amplitude, demonstrating the polarimetric imaging capabilities of RadioAstron at this frequency (see also Pashchenko et al. 2015). Fringes on ground-space baselines were found up to projected baseline distances of 6 Earth's diameters in length, allowing imaging of $0642+449$ with an angular resolution of 0.8 mas - a four-fold improvement over ground VLBI observations at this frequency.

In this paper we present the first polarimetric space VLBI imaging observations at $22 \mathrm{GHz}$, obtained as part of our RadioAstron Key Science Program (KSP), aimed to develop, commission, and exploit the unprecedented high angular resolution polarization capabilities of $R a$ dioAstron to probe the innermost regions of AGN jets and their magnetic fields. A sample of powerful, highly polarized, and $\gamma$-ray emitting blazars is being observed within our KSP, including several quasars, BL Lac objects, and radio galaxies. In this first paper of a series containing our RadioAstron KSP results, we present our observations of BL Lacertae, the eponymous blazar that gives name to the class of BL Lac objects.

The jet of BL Lac is pointing at us with a viewing angle of $\sim 8^{\circ}$ with bulk flow at a Lorentz factor of $\sim 7$ (Jorstad et al. 2005). Previous observations have revealed a multiwavelength outburst, from radio to $\gamma$-rays, triggered by the passing of a bright moving feature through a standing shock associated with the core of the jet (Marscher et al. 2008). Rotation of the optical polarization angle prior to the $\gamma$-ray flare led Marscher et al. (2008) to conclude that the acceleration and collimation zone, upstream of the radio core, is threaded by a helical magnetic field. The existence of a helical magnetic field in BL Lac has also been suggested by Cohen et al. (2015) through the analysis of the MOJAVE monitoring program data. These authors claim that Alfvén waves triggering the formation of superluminal components are excited by changes in the position angle of a recollimation shock (located at a distance from the core of $\sim 0.26$ mas), in a similar way as found in magnetohydrodynamical simulations of relativistic jets threaded by a helical magnetic field (Lind) et al. 1989, Meier 2013).

VLBA monitoring programs at $43 \mathrm{GHz}$ have revealed the existence of a second stationary feature besides the one described previously at 0.26 mas, located at a distance of $\sim 0.1$ mas (Jorstad et al. 2005). Variations in the position angle of these innermost components suggest that the jet in BL Lac may be precessing (Stirling) et al. 2003, Mutel \& Denn 2005).
Faraday rotation analysis reveals a variable rotation measure (RM) in the core of BL Lac, including sign reversals. Observations by Zavala \& Taylor (2003) give -376 rad $\mathrm{m}^{-2}$, while O'Sullivan \& Gabuzda (2009) find $\mathrm{RM}$ values between -1000 and $+240 \mathrm{rad} \mathrm{m}^{-2}$, depending also on the set of frequencies used in the analysis. Significantly larger positive values, between approximately 2000 and $10000 \mathrm{rad} \mathrm{m}^{-2}$, are reported by Stirling et al. (2003) and Jorstad et al. (2007). However rotation measure values for the jet appear very stable, with smaller values in the range between -300 and $\sim 150 \mathrm{rad} \mathrm{m}^{-2}$ (Zavala \& Taylor 2003; O'Sullivan \& Gabuzda 2009, Hovatta et al. 2012).

In Sec. 2 we present the observations and the specific details for the analysis of the space VLBI RadioAstron data; in Sec. 3 we present and analyze the RadioAstron image at $22 \mathrm{GHz}$, whose polarization is analyzed in more detail in Sec. 4. Finally, our conclusions and summary are presented in Sec. 5 .

For a flat Universe with $\Omega_{m}=0.3, \Omega_{\Lambda}=0.7$, and $H_{0}$ $=70 \mathrm{~km} \mathrm{~s}^{-1} \mathrm{Mpc}^{-1}$ (Planck Collaboration et al. 2014), 1 mas corresponds to $1.295 \mathrm{pc}$ at the redshift of BL Lac $(z=0.0686)$, and a proper motion of $1 \mathrm{mas} / \mathrm{yr}$ is equivalent to $4.51 \mathrm{c}$.

\section{OBSERVATIONS AND DATA REDUCTION}

\subsection{RadioAstron space VLBI observations at 22 $\mathrm{GHz}$}

RadioAstron observations of BL Lac at $22.2 \mathrm{GHz}$ (Kband) were performed on 2013 November 10-11 (from 21:30 to 13:00 UT). A total of 26 ground antennas were initially scheduled for the observations, but different technical problems at the sites limited the final number of correlated ground antennas to 15, namely Effelsberg (EF), Metsaehovi (MH), Onsala (ON), Svetloe (SV), Zelenchukskaya (ZC), Medicina (MC), Badary (BD), and VLBA antennas Brewster (BR), Hancock (HN), Kitt Peak (KP), Los Alamos (LA), North Liberty (NL), Owens Valley (OV), Pie Town (PT), and Mauna Kea (MK).

The data were recorded in two polarizations (left and right circularly polarized, LCP and $\mathrm{RCP}$ ), with a total bandwidth of $32 \mathrm{MHz}$ per polarization, split into two intermediate frequency (IF) bands of $16 \mathrm{MHz}$. The SRT data were recorded by the RadioAstron satellite tracking stations (Kardashev et al. 2013; Ford et al. 2014) in Puschino (21:30 - 06:10 UT) and Green Bank (07:3013:00 UT), including some extended gaps required for cooling of the motor drive of the onboard high-gain antenna of the Spektr- $R$ satellite. These gaps were used for the ground-only observations of BL Lac at $15 \mathrm{GHz}$ and $43 \mathrm{GHz}$ (see Sec. 2.2), as well as observations of several calibrator sources.

Correlation of the data was performed using the upgraded version of the DiFX correlator developed at the Max-Planck-Institut für Radiostronomie (MPIfR) in Bonn (Bruni et al. 2014), enabling accurate calibration of the instrumental polarization of the SRT (see also Lobanov et al. 2015, for a more detailed description of the imaging and correlation of RadioAstron observations).

The correlated data were reduced and imaged using a combination of the AIPS and Difmap (Shepherd 1997) software packages. The a priori amplitude calibration was applied using the measured system temperatures for 


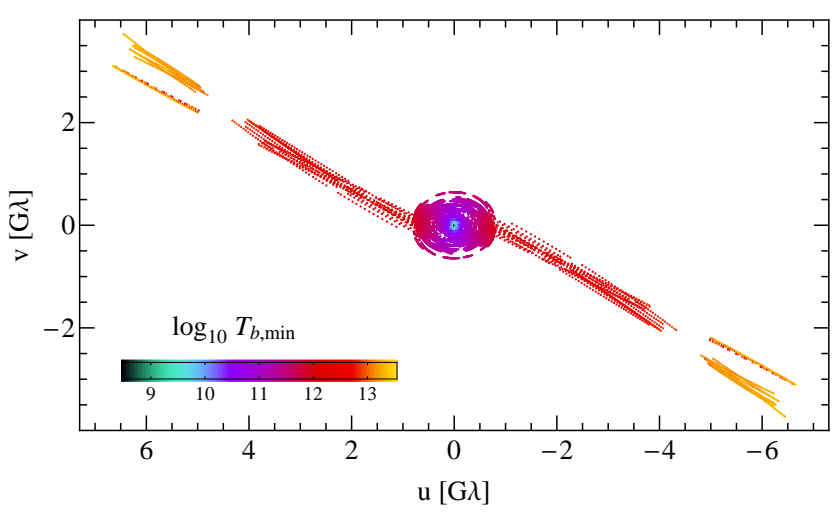

FIG. 1.- Fourier coverage ( $u v$-coverage) of the fringe fitted data (i.e., reliable fringe detections) of the RadioAstron observations of BL Lac on 2013 November $10-11$ at $22 \mathrm{GHz}$. Color marks the lower limit of observed brightness temperature obtained from visibility amplitudes (for details, see discussion in Sect. 3.3.

the ground antennas and the SRT. Sensitivity parameters of the SRT (Kovalev et al. 2014) are measured regularly during maintenance sessions. Parallactic angle corrections were applied to the ground antennas to correct for the feed rotation.

\subsubsection{Fringe fitting}

Fringe fitting of the data was performed by first manually solving for the instrumental phase offsets and single band delays using a short scan during the perigee of the SRT, when the shortest projected ground-space baseline distances (smaller than one Earth's diameter) are obtained, providing the best fringe solutions for the space antenna. These solutions were applied before performing a global fringe search for the delays and rates of the ground array only.

Once the ground antennas were fully calibrated, they were coherently combined to improve the fringe detection sensitivity of the SRT (Kogan 1996). This baseline stacking was carried out setting $\operatorname{DPARM}(1)=3$ in AIPS's FRING task, performing also an exhaustive baseline search, and combining both polarizations and IFs to improve the sensitivity. Progressively longer solution intervals were used for the fringe search, from one minute to maintain coherence of the signal during the acceleration of the space craft in the perigee, to four minutes to increase the sensitivity on the longer baselines to the SRT.

The phasing of a group of ground-based antennas allowed us to obtain reliable ground-space fringe detections up to projected baselines of 7.9 Earth's diameters $\left(D_{\mathrm{E}}\right)$ in length, covering the duration of the experiment within which Puschino was used as the tracking station. No further fringes were obtained to the space craft once the tracking station changed to Green Bank, which is presumably due to a difference in clock setting between the two tracking stations. These were searched for by introducing trial clock offsets for the Green Bank tracking station, and performing new test correlations with a larger fringe-search window of up to 1024 channels and $0.1 \mathrm{sec}$ of integration time in width. However, no further fringes were detected to the SRT. We also note that 1.5 hours passed between the last Puschino scan and the first Green Bank scan, thus increasing the space baseline length and perhaps reducing the correlated flux den-
TABLE 1

InStRumentAl POLARIZATION (D-TERMS) AT $22 \mathrm{GHz}$

\begin{tabular}{|c|c|c|c|c|}
\hline \multirow[t]{2}{*}{ Antenna } & \multicolumn{2}{|c|}{$\mathrm{RCP}$} & \multicolumn{2}{|c|}{ LCP } \\
\hline & $\begin{array}{r}m \\
{[\%]}\end{array}$ & $\begin{array}{c}\chi \\
\left.{ }^{\circ}\right]\end{array}$ & $\begin{array}{r}m \\
{[\%]}\end{array}$ & $\begin{array}{c}\chi \\
{\left[{ }^{\circ}\right]}\end{array}$ \\
\hline \multirow[t]{2}{*}{ SRT } & $9.3 \pm 0.5$ & $21 \pm 5$ & $4.5 \pm 0.3$ & $72 \pm 5$ \\
\hline & $8.8 \pm 0.8$ & $20 \pm 4$ & $4.4 \pm 0.2$ & $68 \pm 8$ \\
\hline \multirow[t]{2}{*}{$\mathrm{BR}$} & $1.4 \pm 0.7$ & $-73 \pm 18$ & $0.8 \pm 0.4$ & $-165 \pm 22$ \\
\hline & $1.4 \pm 0.7$ & $-86 \pm 23$ & $0.7 \pm 0.3$ & $-196 \pm 24$ \\
\hline \multirow[t]{2}{*}{$\mathrm{EF}$} & $9.9 \pm 0.7$ & $-91 \pm 4$ & $8.1 \pm 0.5$ & $-126 \pm 7$ \\
\hline & $9.3 \pm 0.8$ & $-98 \pm 3$ & $7.5 \pm 0.3$ & $-130 \pm 6$ \\
\hline \multirow[t]{2}{*}{$\mathrm{HN}$} & $2.3 \pm 0.2$ & $174 \pm 16$ & $2.2 \pm 0.6$ & $90 \pm 6$ \\
\hline & $2.2 \pm 0.4$ & $149 \pm 14$ & $2.0 \pm 0.8$ & $85 \pm 11$ \\
\hline \multirow[t]{2}{*}{ KP } & $1.1 \pm 0.3$ & $-160 \pm 8$ & $1.1 \pm 0.4$ & $-167 \pm 12$ \\
\hline & $0.9 \pm 0.4$ & $-182 \pm 5$ & $1.0 \pm 0.3$ & $-194 \pm 8$ \\
\hline \multirow[t]{2}{*}{ LA } & $2.4 \pm 0.6$ & $-62 \pm 7$ & $1.0 \pm 0.4$ & $-124 \pm 8$ \\
\hline & $2.7 \pm 0.7$ & $-75 \pm 6$ & $0.8 \pm 0.6$ & $-126 \pm 11$ \\
\hline \multirow[t]{2}{*}{ NL } & $3.6 \pm 0.5$ & $-43 \pm 5$ & $3.8 \pm 0.2$ & $-106 \pm 9$ \\
\hline & $3.1 \pm 0.6$ & $-43 \pm 7$ & $4.0 \pm 0.4$ & $-104 \pm 8$ \\
\hline \multirow[t]{2}{*}{ OV } & $2.0 \pm 0.8$ & $118 \pm 8$ & $2.3 \pm 0.4$ & $18 \pm 7$ \\
\hline & $2.2 \pm 0.8$ & $94 \pm 6$ & $2.7 \pm 0.4$ & $13 \pm 11$ \\
\hline \multirow[t]{2}{*}{$\mathrm{PT}$} & $1.4 \pm 0.3$ & $-85 \pm 11$ & $2.0 \pm 0.5$ & $-74 \pm 9$ \\
\hline & $1.4 \pm 0.4$ & $-77 \pm 12$ & $2.0 \pm 0.6$ & $-65 \pm 12$ \\
\hline \multirow[t]{2}{*}{$\mathrm{MH}$} & $1.9 \pm 0.9$ & $-116 \pm 8$ & $8.2 \pm 1.3$ & $-41 \pm 9$ \\
\hline & $2.9 \pm 0.8$ & $-152 \pm 6$ & $5.5 \pm 0.9$ & $-57 \pm 8$ \\
\hline \multirow[t]{2}{*}{$\mathrm{ON}$} & $4.1 \pm 0.8$ & $-43 \pm 4$ & $5.3 \pm 0.5$ & $-87 \pm 7$ \\
\hline & $4.2 \pm 0.9$ & $-38 \pm 8$ & $5.2 \pm 0.6$ & $-82 \pm 12$ \\
\hline \multirow[t]{2}{*}{ SV } & $4.8 \pm 0.3$ & $179 \pm 7$ & $4.1 \pm 0.5$ & $-2 \pm 5$ \\
\hline & $4.7 \pm 0.2$ & $178 \pm 10$ & $4.0 \pm 0.3$ & $0 \pm 4$ \\
\hline \multirow[t]{2}{*}{$\mathrm{ZC}$} & $6.1 \pm 0.7$ & $162 \pm 13$ & $8.3 \pm 0.8$ & $168 \pm 9$ \\
\hline & $8.3 \pm 0.6$ & $111 \pm 10$ & $6.9 \pm 0.9$ & $165 \pm 5$ \\
\hline \multirow[t]{2}{*}{$\mathrm{MC}$} & $0.9 \pm 0.7$ & $109 \pm 10$ & $6.1 \pm 0.6$ & $41 \pm 8$ \\
\hline & $2.6 \pm 0.9$ & $87 \pm 11$ & $5.5 \pm 0.9$ & $42 \pm 11$ \\
\hline \multirow[t]{2}{*}{ MK } & $3.3 \pm 0.4$ & $-64 \pm 8$ & $2.9 \pm 0.3$ & $-135 \pm 5$ \\
\hline & $3.5 \pm 0.6$ & $-67 \pm 12$ & $2.9 \pm 0.5$ & $-130 \pm 9$ \\
\hline \multirow[t]{2}{*}{$\mathrm{BD}$} & $7.7 \pm 0.8$ & $-99 \pm 7$ & $6.4 \pm 0.6$ & $-179 \pm 6$ \\
\hline & $8.1 \pm 0.9$ & $-103 \pm 8$ & $6.7 \pm 0.8$ & $-178 \pm 9$ \\
\hline
\end{tabular}

Notes. Listed values correspond to the fractional amplitude, $m$, and phase, $\chi$, of the instrumental polarization for each antenna and polarization in the first (top row) and second (bottom row) IF.

sity below the sensitivity threshold. The obtained fringefitted data visibility coverage of the Fourier domain ( $u v$ coverage) is shown in Fig. 1.

After the fringe fitting the delay difference between the two polarizations was corrected using AIPS's task RLDLY, and a complex bandpass function was solved for the receiver.

\subsubsection{Polarization calibration}

The instrumental polarization (D-terms) was obtained using AIPS's task LPCAL (Leppanen et al. 1995). Table 1 lists the instrumental polarization derived for each telescope, with Effelsberg as the reference antenna. Errors in the instrumental polarization were estimated from the dispersion in the values obtained by performing independent data reductions while using different reference antennas, as well as comparison with values obtained for calibrator sources $(2021+614$ and $1823+568)$. Estimated values for the ground antennas are also subject to antenna performance and weather conditions at the sites, which in some cases may lead to larger than usual instrumental polarization values. Amplitude and phase stability across the two IFs confirms the reliability of the 


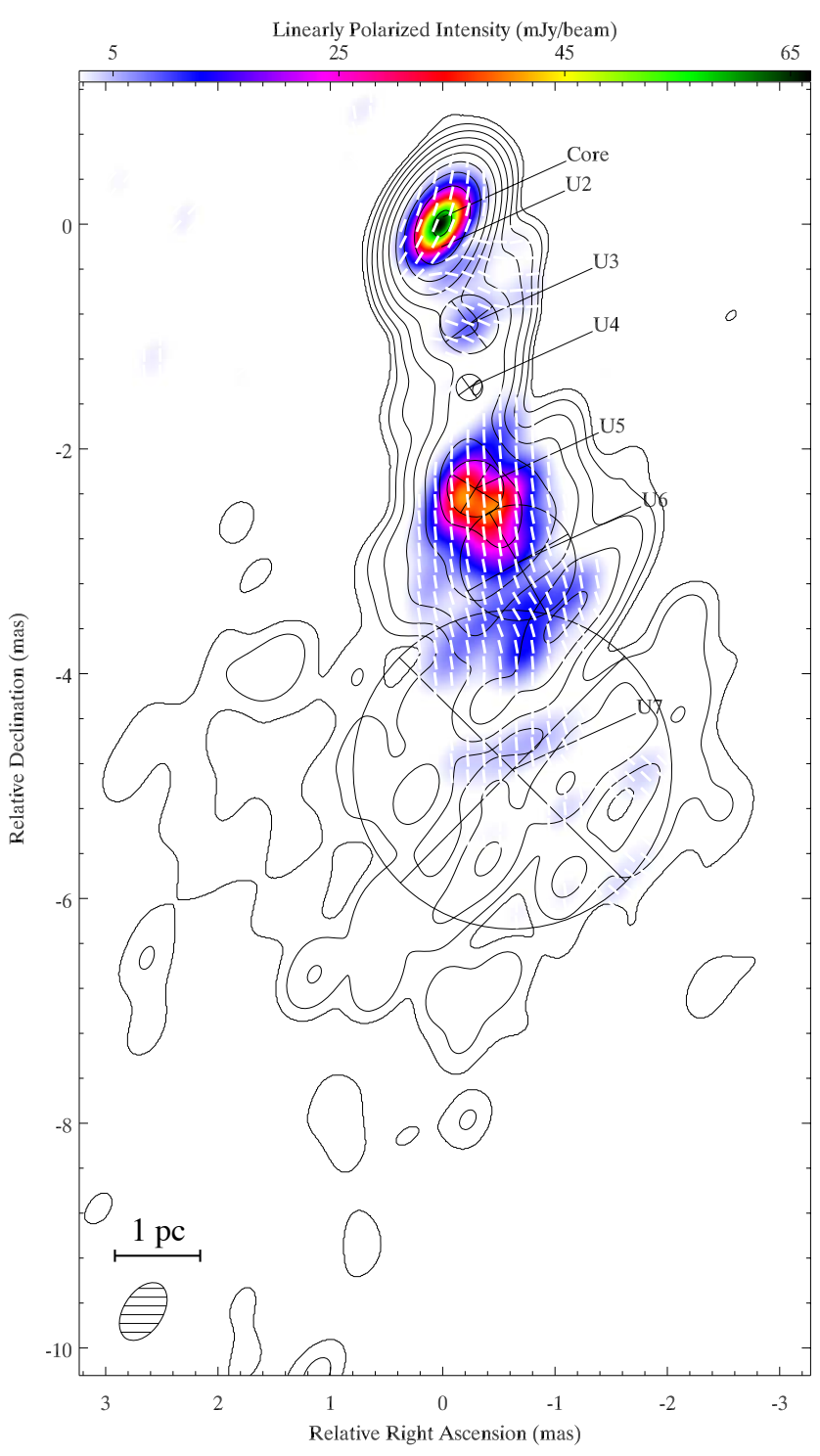

Fig. 2.- Ground-array $15 \mathrm{GHz}$ observations of BL Lac on 2013 November 10 . Total intensity contours are plotted at $0.05,0.11$, $0.24,0.57,1.32,3.08,7.16,16.64,38.70$, and $90 \%$ of the peak intensity at $3.31 \mathrm{Jy} /$ beam. Linearly polarized intensity is shown in colors starting at $2 \mathrm{mJy} / \mathrm{beam}$, and bars indicate the EVPA. Synthesized beam FWHM is $0.57 \times 0.35$ mas at a position angle of $-33^{\circ}$. Model fit components are also shown overlaid, indicating their position and angular size (see also Table 2).

estimated values.

Instrumental polarization of the SRT at $22 \mathrm{GHz}$ is within $9 \%$ ( $5 \%$ for LCP), and remarkably consistent across the two IFs, demonstrating its robust polarization capabilities for RadioAstron imaging observations at its highest observing frequency of $22 \mathrm{GHz}$.

Absolute calibration of the electric vector position angle (EVPA) was obtained from comparison with simultaneous single dish observations at the Effelsberg telescope of our target and calibrator sources. We estimate the error in the EVPA calibration to lie between $5^{\circ}$ and $10^{\circ}$.

\subsection{Ground-array observations at $15 \mathrm{GHz}$ and $43 \mathrm{GHz}$}

Simultaneous ground-only observations of BL Lac at 15.4 GHz and $43.1 \mathrm{GHz}$ were obtained during gaps in the RadioAstron observations. Participating antennas were Effelsberg and VLBA antennas BR, HN, KP, LA,

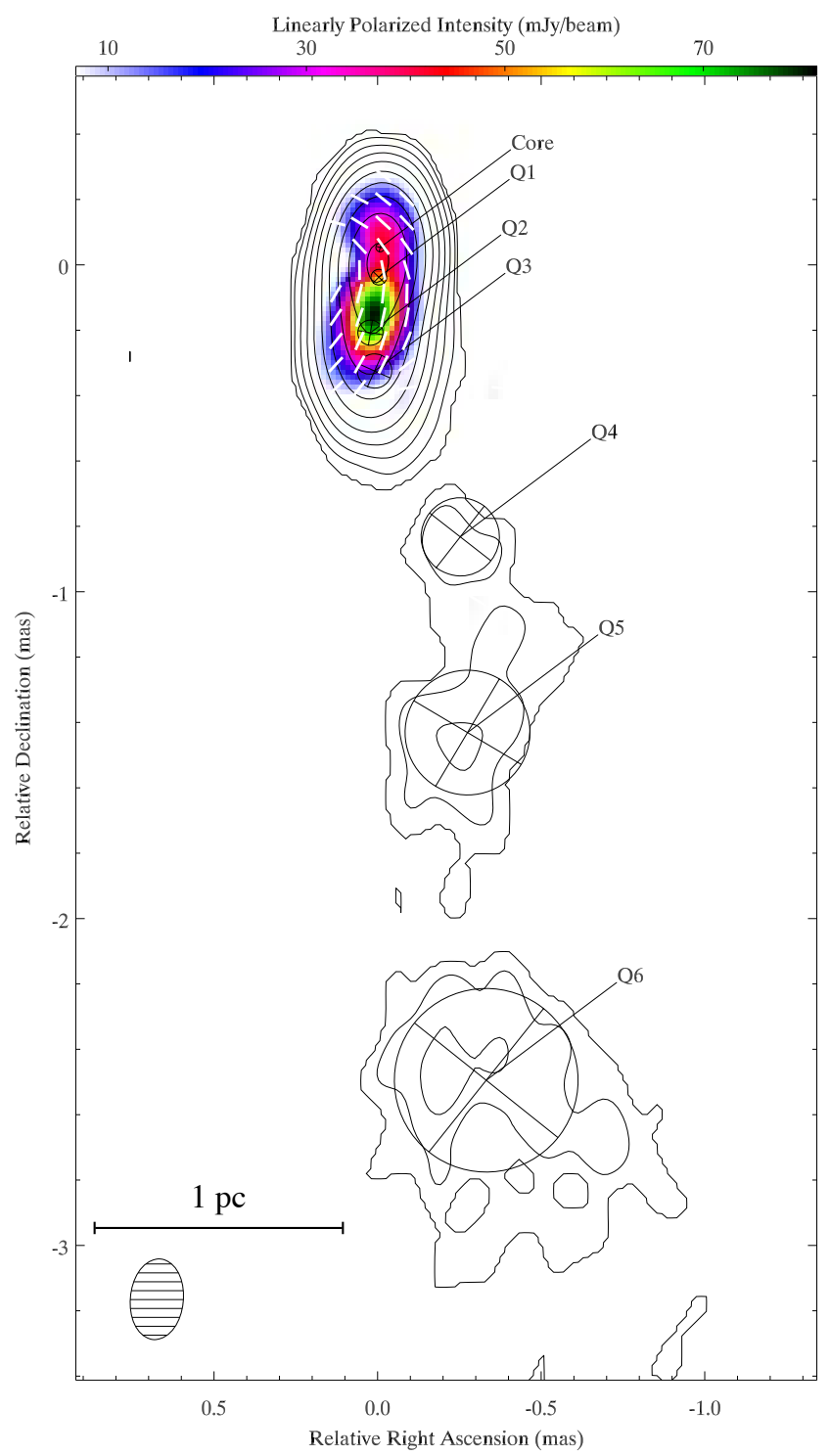

Fig. 3.- VLBA-BU-BLAZAR image of BL Lac at $43 \mathrm{GHz}$ obtained in 2013 November 18 . Total intensity contours are plotted at $0.25,0.48,0.92,1.78,3.42,6.57,12.65,24.33,46.79$, and $90 \%$ of the peak at $2.7 \mathrm{Jy} /$ beam. Linearly polarized intensity is shown in colors starting at $6.8 \mathrm{mJy} / \mathrm{beam}$, and bars indicate the EVPA. Synthesized beam FWHM is $0.25 \times 0.16$ mas at a position angle of $-5^{\circ}$. Model fit components are also shown overlaid, indicating their position and angular size (see also Table 2).

NL, OV, and PT. However, at $43 \mathrm{GHz}$ no fringes were detected on the intercontinental baselines with Effelsberg due to technical problems, severely limiting the sensitivity and angular resolution, in fact preventing the detection of polarization at this frequency. For this reason we have used instead the $43 \mathrm{GHz}$ data from the VLBABU-BLAZAR ${ }^{11}$ monitoring program, performed on 2013 November 18th, only one week after our observations.

Calibration of the $15 \mathrm{GHz}$ data follows that outlined previously in Sec. 2.1, except for the particular steps related more directly to the space VLBI observations, such as the phasing of the ground-array during the fringe fitting. Calibration of the absolute orientation of the EVPAs was also performed by comparison with Effelsberg

11 see https://www.bu.edu/blazars/VLBAproject.html 


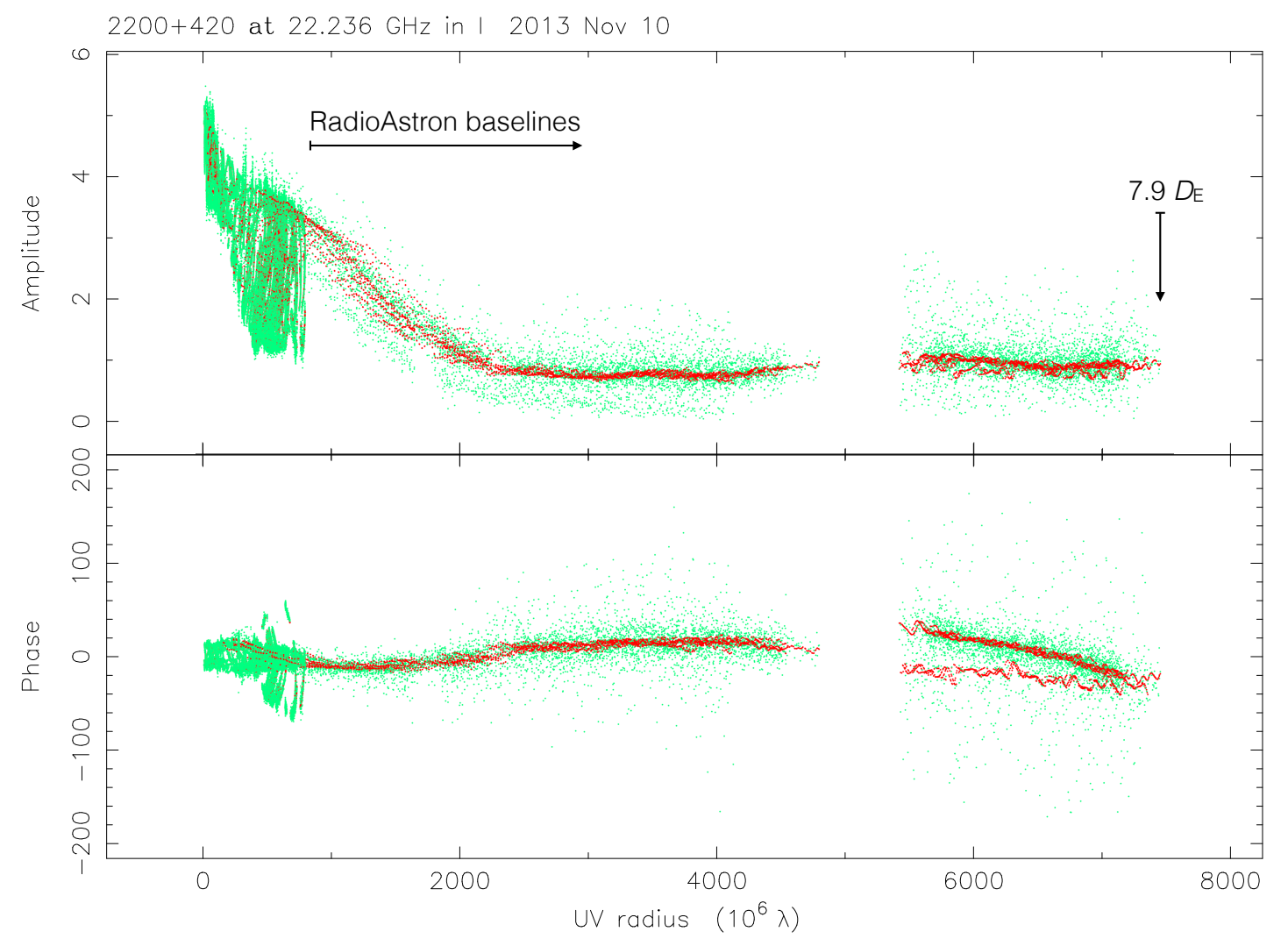

FIG. 4.- Self-calibrated visibility amplitudes and phases as a function of uv-distance of the RadioAstron observations of BL Lac on 2013 November 10-11 at $22 \mathrm{GHz}$. Overplotted in red is the fit to these data of the CLEAN model obtained from the hybrid mapping. Reliable space-ground fringe detections were obtained up to a projected baseline distance of 7.9 Earth's diameters.

single dish observations of BL Lac $\left(S_{15 \mathrm{GHz}}=5.57 \pm 0.23\right.$ Jy, $\left.m=3.44 \pm 0.3 \%, \mathrm{EVPA}=3.7 \pm 1.1^{\circ}\right)$, with an estimated error of $5^{\circ}$ to $7^{\circ}$.

Figures 2 and 3 show the $15 \mathrm{GHz}$ and $43 \mathrm{GHz}$ images of BL Lac obtained with the ground array. To characterize the emission structure we have performed a fit of the complex visibilities by a set of components with circular Gaussian brightness distributions, listed in Table 2.

\section{SPACE VLBI POLARIMETRIC IMAGES OF BL LAC AT $21 \mu$ AS ANGULAR RESOLUTION}

Fully calibrated RadioAstron data were exported to Difmap and imaged using the standard hybrid imaging and self-calibration techniques. Self-calibrated Stokes I visibility amplitudes and phases as a function of Fourier spacing ( $u v$ distance) and CLEAN model fit to these data are shown in Fig. 4. Space VLBI fringes to the SRT extend the projected baseline spacing up to $7.9 D_{\mathrm{E}}$, increasing accordingly the angular resolution with respect to that provided by ground-based arrays. However the large eccentricity of the SRT orbit (see Fig. 1) leads to a highly elliptical observing beam.

RadioAstron space VLBI polarimetric images of BL Lac are shown in Fig. 5 for three different visibility weights: natural, uniform, and "super" uniform (in which the gridding weights are not scaled by the visibility amplitude errors). The weights of the longest space VLBI visibilities are therefore increasingly higher from natural to super uniform weightings, consequently yielding higher angular resolutions, albeit with lower image sensitivities. The super uniform weighting image yields an angular resolution of $21 \mu$ as (along the minor axis of the restoring beam), which to our knowledge corresponds to the highest achieved to date. For an estimated black hole mass of $\sim 1.6 \times 10^{8} \mathrm{M}_{\odot}$ ( Woo \& Urry 2002), where $\mathrm{M}_{\odot}$ is the mass of the Sun, this corresponds to a linear resolution of $\sim 1800$ Schwarzschild radii at the BL Lac distance.

The images in Fig. 5 show the familiar radio continuum emission structure of BL Lac, dominated by the core and a jet that extends to the south. Conveniently, the highest angular resolution provided by the groundspace baselines is obtained nearly along the jet direction, allowing close examination of the innermost structure of the jet. As can be better distinguished in the uniform (Fig. 5b) and super uniform (Fig. 5k) images, the total intensity images reveal a bent structure in the innermost 0.5 mas region. The linearly polarized images clearly distinguish two components in this region, as well as in the jet area at $\sim 3$ mas from the core.

\subsection{Stationary components in the innermost 0.5 mas region}

Previous high angular resolution monitoring programs of BL Lac systematically show the presence of two stationary features close to the core (Stirling et al. 2003, Jorstad et al. 2005; Mutel \& Denn 2005). In particular, Jorstad et al. (2005) report, through an analysis of a sequence of 17 bimonthly VLBA observations at $43 \mathrm{GHz}$, two stationary components, labeled A1 and A2, located at a mean distance from the core (position angle) of 0.10 mas and 0.29 mas $\left(-160^{\circ}\right.$ and $\left.-159^{\circ}\right)$, respectively. 


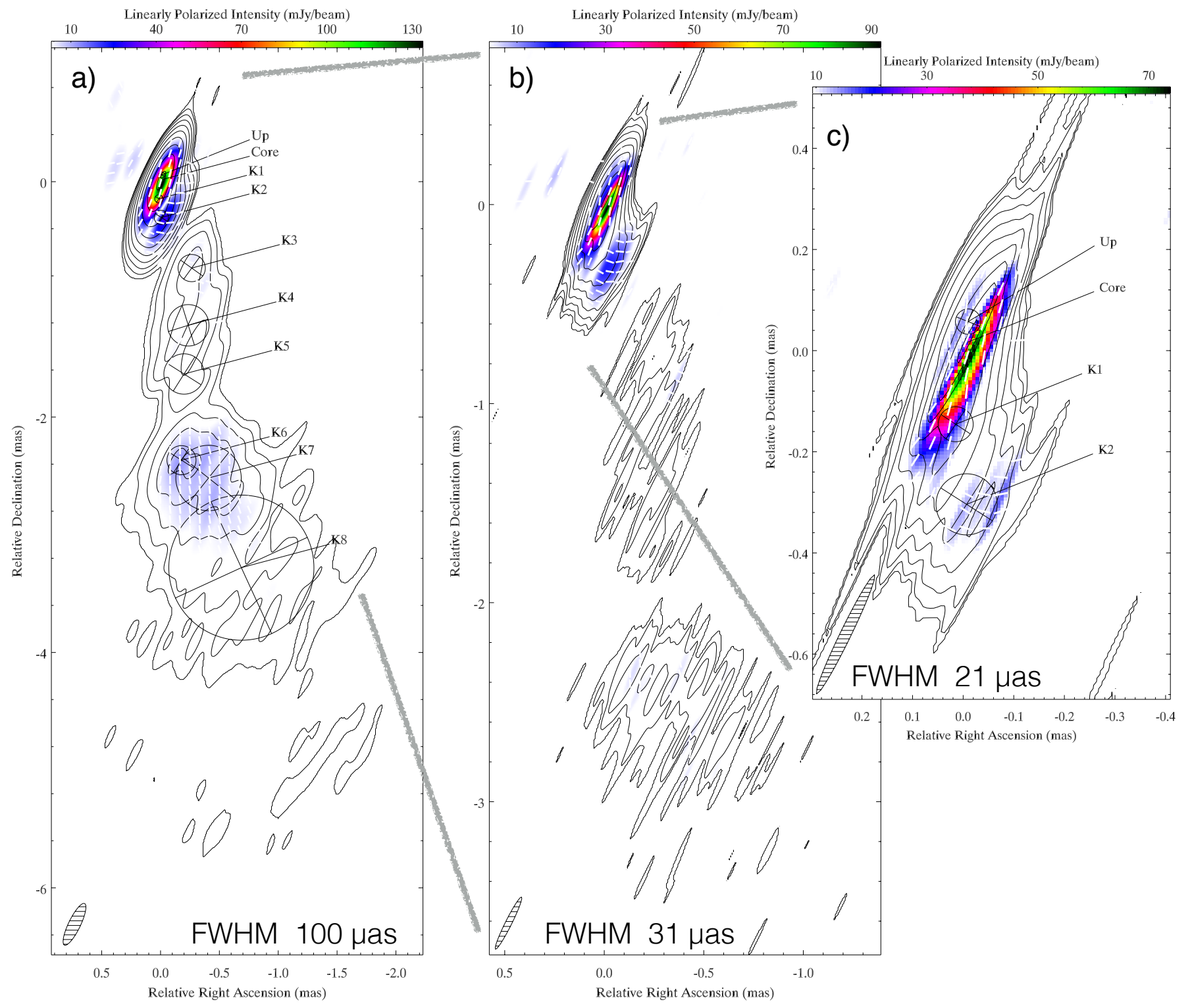

FIG. 5.- RadioAstron polarimetric space VLBI images of BL Lac obtained in 2013 November 10-11 at 22 GHz. From left to right, images are obtained with natural (a), uniform (b), and "super"-uniform (c) weightings. Total intensity contours are plotted at $0.08,0.17,0.36,0.80$, $1.76,3.86,8.49,18.65,40.97$, and $90 \%(0.2,0,4,0.78,1.54,3.04,5.99,11.79,23.22,45,71$, and $90 \% ; 0.76,1.3,2.21,3.75,6.37,10.81,18.37$, $31.2,23$, and $90 \%)$ of the peak intensity at $2.48(1.48 ; 1.23) \mathrm{Jy}$ /beam for the natural weighted image (uniform; "super"-uniform). Linearly polarized intensity is shown in colors starting at (a) $3.1 \mathrm{mJy} / \mathrm{beam}$, (b) $3.8 \mathrm{mJy} / \mathrm{beam}$, and (c) $9.4 \mathrm{mJy} / \mathrm{beam}$, and white bars indicate the (uncorrected for Faraday rotation) EVPAs. Synthesized beams are (a) $0.392 \times 0.100$ mas, (b) $0.292 \times 0.031$ mas, and (c) $0.261 \times 0.021$ mas at a common position angle of $-26^{\circ}$.

These can be associated with components C2 and C3 in Mutel \& Denn (2005), respectively, obtained from independent $43 \mathrm{GHz}$ VLBA observations.

More recently, Cohen et al. (2014, 2015) present an analysis of more than a decade of $15 \mathrm{GHz}$ VLBA observations of BL Lac from the MOJAVE monitoring program. These observations confirm the existence of a stationary component, labeled C7 by these authors, located at a mean distance from the core of 0.26 mas and at a position angle of $-166^{\circ} 6$, in agreement with component A2 reported by Jorstad et al. (2005).

This is also corroborated by our $15 \mathrm{GHz}$ and $43 \mathrm{GHz}$ observations (see Table 2 and Figs. 2 and 3), in which components U2 and Q2 would correspond to previously identified components $\mathrm{A} 2$ and $\mathrm{C} 7$, and component Q1 to A1.
Our measured position angles for the two stationary features (Q1 and Q2/U2) are slightly offset to the east by $\sim 20^{\circ}$ with respect to the main values published by Jorstad et al. (2005) and Cohen et al. (2014). This may be associated with the jet precession reported by Stirling et al. (2003) and Mutel \& Denn (2005), leading to a swing in the position angle of the innermost components. A similar variation in the position angle of component U2 is seen in MOJAVE observations by Cohen et al. (2014).

\subsection{Evidence for emission upstream of the core}

Model fitting of the innermost structure in our $R a$ dioAstron observations, listed in Table 2 and plotted in Fig. 5. shows two close components near the core region, as well as two other components within the innermost 0.5 mas region. The fitted circular Gaussian components 
TABLE 2

Gaussian model fits For the 22 GHz RAdioAstron AND Ground-ARray data AT 43 AND 15 GHz

\begin{tabular}{|c|c|c|c|c|c|c|c|}
\hline Comp. & $\begin{array}{l}\text { Flux } \\
(\mathrm{mJy})\end{array}$ & $\begin{array}{l}\text { Distance } \\
\text { (mas) }\end{array}$ & $\begin{array}{l}\text { PA } \\
\left({ }^{\circ}\right)\end{array}$ & $\begin{array}{c}\text { Size } \\
(\mathrm{mas}) \\
\end{array}$ & $\begin{array}{l}\mathrm{T}_{\mathrm{b}} \\
(\mathrm{K})\end{array}$ & $\begin{array}{c}m \\
(\%)\end{array}$ & $\begin{array}{c}\text { EVPA } \\
\left({ }^{\circ}\right)\end{array}$ \\
\hline \multicolumn{8}{|c|}{ RadioAstron $22 \mathrm{GHz}$} \\
\hline Up & $1578 \pm 72$ & $0.041 \pm 0.003$ & $-1 \pm 4$ & $0.050 \pm 0.003$ & $(1.56 \pm 0.26) \times 10^{12}$ & $4.0 \pm 0.4$ & $-17 \pm 1$ \\
\hline Core & $802 \pm 43$ & & $\ldots$ & $<0.01$ & $>2.0 \times 10^{13}$ & $4.8 \pm 0.4$ & $-16 \pm 1$ \\
\hline $\mathrm{K} 1$ & $1128 \pm 37$ & $0.164 \pm 0.004$ & $172 \pm 1$ & $0.067 \pm 0.003$ & $(5.82 \pm 0.70) \times 10^{11}$ & $5.7 \pm 0.5$ & $-17 \pm 1$ \\
\hline $\mathrm{K} 2$ & $578 \pm 12$ & $0.320 \pm 0.008$ & $179 \pm 1$ & $0.122 \pm 0.004$ & $(9.79 \pm 0.85) \times 10^{10}$ & $8.6 \pm 2.0$ & $84 \pm 5$ \\
\hline K3 & $79 \pm 12$ & $0.79 \pm 0.03$ & $-161 \pm 2$ & $0.231 \pm 0.012$ & $(3.64 \pm 0.93) \times 10^{9}$ & $\ldots$ & .. \\
\hline K4 & $111 \pm 13$ & $1.26 \pm 0.04$ & $-170 \pm 1$ & $0.352 \pm 0.018$ & $(2.22 \pm 0.49) \times 10^{9}$ & & $\ldots$ \\
\hline K5 & $70 \pm 13$ & $1.66 \pm 0.05$ & $-173 \pm 2$ & $0.343 \pm 0.017$ & $(1.47 \pm 0.42) \times 10^{9}$ & & \\
\hline K6 & $108 \pm 12$ & $2.39 \pm 0.02$ & $-176 \pm 1$ & $0.247 \pm 0.012$ & $(4.39 \pm 0.91) \times 10^{9}$ & $20.8 \pm 2.4$ & $4 \pm 4$ \\
\hline K7 & $283 \pm 14$ & $2.57 \pm 0.04$ & $-171 \pm 1$ & $0.556 \pm 0.056$ & $(2.26 \pm 0.57) \times 10^{9}$ & $26.2 \pm 6.7$ & $8 \pm 3$ \\
\hline K8 & $214 \pm 15$ & $3.37 \pm 0.11$ & $-168 \pm 2$ & $1.23 \pm 0.12$ & $(3.48 \pm 0.92) \times 10^{8}$ & $\ldots$ & $\ldots$ \\
\hline \multicolumn{8}{|c|}{ VLBA-BU-BLAZAR ground array at $43 \mathrm{GHz}$} \\
\hline Core & $1575 \pm 72$ & & & $0.026 \pm 0.005$ & $(1.48 \pm 0.63) \times 10^{12}$ & $1.7 \pm 0.2$ & $37 \pm 6$ \\
\hline Q1 & $1373 \pm 65$ & $0.091 \pm 0.005$ & $177 \pm 8$ & $0.048 \pm 0$ & $(3.92 \pm 1.00) \times$ & $2.0 \pm 0.3$ & $7 \pm 4$ \\
\hline Q2 & $86 \pm 32$ & & $173 \pm 1$ & $0.077 \pm 0$ & $(8.68 \pm 1.93) \times 1$ & $5.4 \pm 0.1$ & $-18 \pm 2$ \\
\hline Q3 & $402 \pm 18$ & $0.378 \pm 0$ & $177 \pm 1$ & $0.105 \pm 0$ & $(2.40 \pm 0.52) \times 10^{10}$ & $3.1 \pm 0.8$ & $-29 \pm 4$ \\
\hline Q4 & $50 \pm 10$ & & $-165 \pm 5$ & $0.239 \pm 0$ & $(5.79 \pm 1.74) \times 10^{8}$ & & \\
\hline Q5 & $100 \pm 11$ & $1.51 \pm 0$ & $-170 \pm 3$ & $0.382 \pm 0$ & $(4.52 \pm 0.95) \times 10^{8}$ & & \\
\hline Q6 & $209 \pm 13$ & $2.57 \pm 0.09$ & $-173 \pm 2$ & $0.562 \pm 0.028$ & $(4.34 \pm 0.70) \times 10^{8}$ & $\ldots$ & \\
\hline \multicolumn{8}{|c|}{ Ground array $15 \mathrm{GHz}$} \\
\hline Core & $2705 \pm 124$ & & & $<0.06$ & $>7.9 \times 10^{12}$ & $2.0 \pm 0.1$ & $-20 \pm 1$ \\
\hline $\mathrm{U} 2$ & $1193 \pm 60$ & $0.266 \pm 0.019$ & $177 \pm 5$ & $<0.06$ & $>3.5 \times 10^{12}$ & $1.5 \pm 0.1$ & $-30 \pm 1$ \\
\hline $\mathrm{U} 3$ & $266 \pm 23$ & $0.982 \pm 0.025$ & $-166 \pm 2$ & $0.523 \pm 0.026$ & $(5.03 \pm 0.94) \times 10^{9}$ & $5.5 \pm 1.7$ & $67 \pm 3$ \\
\hline $\mathrm{U} 4$ & $84 \pm 13$ & $1.53 \pm 0.02$ & $-171 \pm 1$ & $0.235 \pm 0.012$ & $(7.87 \pm 2.00) \times 10^{9}$ & 等 & \\
\hline U5 & $462 \pm 32$ & $2.43 \pm 0.02$ & $-173 \pm 1$ & $0.503 \pm 0.025$ & $(9.44 \pm 1.59) \times 10^{9}$ & $18.9 \pm 4.0$ & $5 \pm 1$ \\
\hline $\mathrm{U} 6$ & $332 \pm 27$ & $3.15 \pm 0.04$ & $-167 \pm 1$ & $1.03 \pm 0.05$ & $(1.62 \pm 0.29) \times 10^{9}$ & $27.0 \pm 18$ & $16 \pm 8$ \\
\hline U7 & $391 \pm 33$ & $4.96 \pm 0.12$ & $-173 \pm 2$ & $2.84 \pm 0.14$ & $(2.52 \pm 0.46) \times 10^{8}$ & $\ldots$ & - \\
\hline
\end{tabular}

Notes. Tabulated data correspond to: component's label; flux density; distance and position angle from the core; size; observed brightness temperature, degree of linear polarization; and electric vector position angle, uncorrected for Faraday rotation (see Sec. 4).

provide an accurate representation of the jet emission, yielding a residual map (uniform weighting) with a rms of $1.4 \mathrm{mJy} / \mathrm{beam}$ and minimum and maximum residuals of $-45 \mathrm{mJy} /$ beam and $53 \mathrm{mJy} /$ beam, respectively. Allowing for elliptical Gaussian components provides very similar fitted values as those listed in Table 2 with no significant improvement in the residuals.

We can tentatively identify components $\mathrm{K} 1$ and K2 with the previously discussed stationary features Q1 and Q2 (U2). However identification of the two-component structure in the core area requires a more detailed analysis of the evolution of the innermost structure of the jet.

For this we have performed model fitting of the $43 \mathrm{GHz}$ VLBA images from the VLBA-BU-BLAZAR program extending our analysis to cover the period between 2013 December and 2014 June, comprising a total of five more epochs with a cadence of roughly one month. The obtained model fits are listed in Table 3 , and Fig. 7 plots the component's distance from the core versus time. Stationary components Q1 and Q2 are detected at all epochs. Analysis of their flux densities show that Q1 becomes unusually bright (2.1 Jy) on 2013 December 16, followed by a similar increase in flux density of Q2 (1.1 Jy) on 2014 January 19. This could be associated with the passing of a new component, M1, through the standing features, leading to a brief increase in their flux densities (e.g., Gómez et al. 1997). Component M1 is identified in the last two epochs as a weak knot beyond 0.5 mas from the core. The estimated apparent speed for this new compo- nent is $7.9 \pm 0.3 c(1.76 \pm 0.06 \mathrm{mas} / \mathrm{yr})$, in agreement with values previously found in BL Lac (Jorstad et al. 2005), giving an ejection date of $2013.89 \pm 0.05$, or 2013 November 23 ( \pm 18 days), in coincidence with our RadioAstron observations within the errors.

Considering the measured proper motion of the new component and the estimated time of crossing through the $43 \mathrm{GHz}$ core, we can estimate that component M1 should be placed $\sim 50 \mu$ as upstream of the core in our RadioAstron image, or slightly smaller if we account for some initial acceleration. Based on this, we consider that the core of the jet in our RadioAstron observations corresponds to the unresolved component labeled "Core", upstream of which component "Up" would correspond to component M1 identified also at $43 \mathrm{GHz}$. Evidence for emission upstream the core is also found by looking at the $43 \mathrm{GHz}$ polarization image (see Fig. 3), which shows polarized emission to the north with a different orientation of the polarization vectors than the remaining core area.

We note that the appearance of new superluminal components upstream of the core in BL Lac has been previously reported by Marscher et al. (2008), associated with a multi-wavelength outburst. Similarly, the radio, optical, and $\gamma$-ray light curves (see the VLBA-BU-BLAZAR web pag $6^{12}$ show a flare at the end of 2013 , close to our RadioAstron observations.

\footnotetext{
12 https://www.bu.edu/blazars/VLBA_GLAST/bllac.html
} 


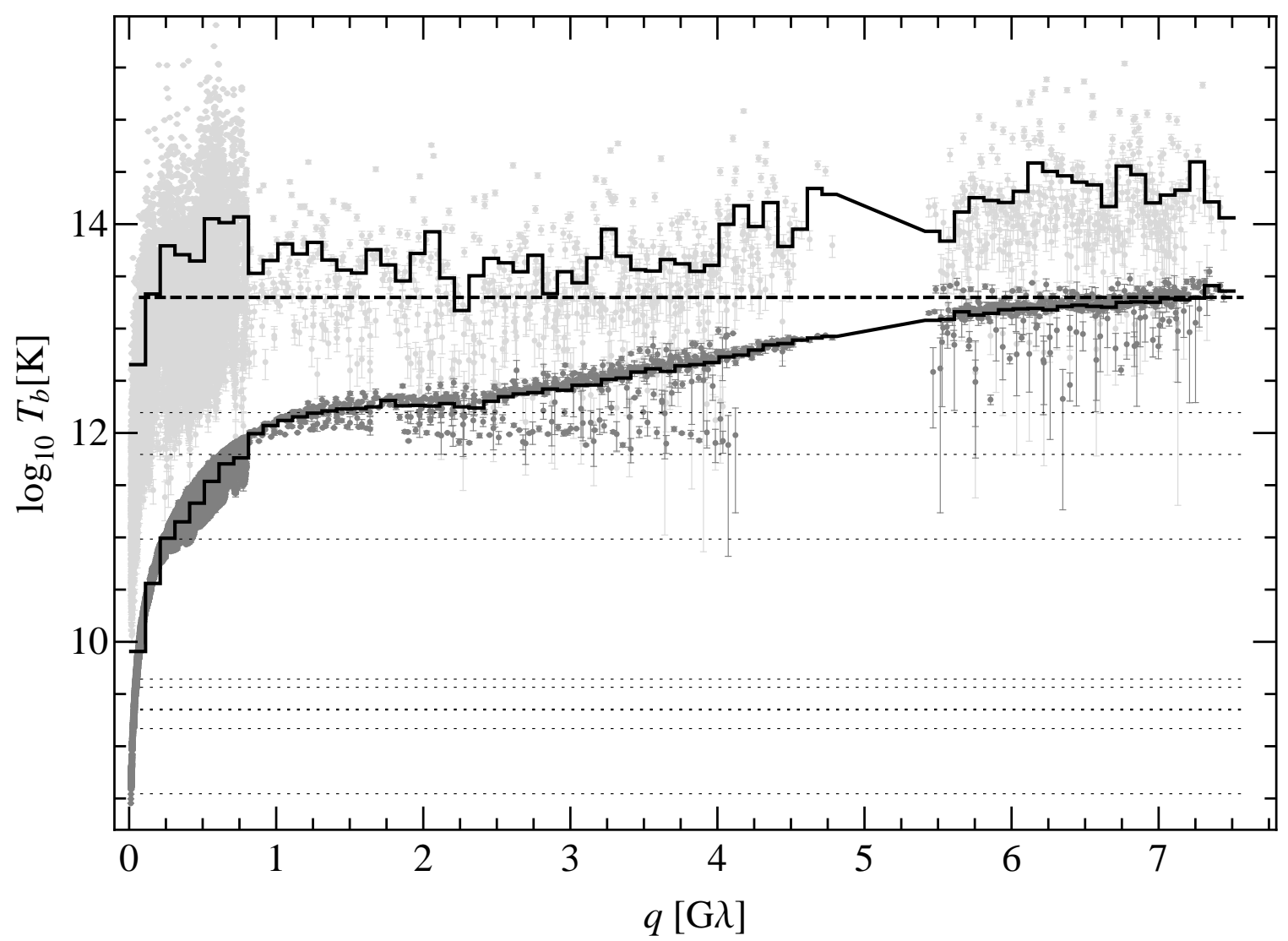

FIG. 6.- Visibility-based estimates of the observed brightness temperature in BL Lac, calculated from the RadioAstron data at 22 GHz. Data points represent the minimum (dark gray), $T_{\mathrm{b}, \text { min }}$, and maximum (light gray), $T_{\mathrm{b}, \text { max }}$, brightness temperatures derived from individual visibilities measured at different $u v$ distances, $q$ (Lobanov 2015). The two-dimensional distribution of $T_{\mathrm{b}}$, min is also presented in Fig. 1 with a color wedge. The histograms show the respective brightness temperatures averaged over bins of $0.1 \mathrm{G} \lambda$ in size. The thick dashed line indicates the lower limit on brightness temperature, $T_{\mathrm{b} \text {,mod }}>2 \times 10^{13} \mathrm{~K}$, derived for the core component of the source structure described in Table 2 The dotted lines indicate the respective brightness temperatures of the rest of the model components. The minimum brightness temperature, $T_{\mathrm{b}, \min }=1.5 \times 10^{13} \mathrm{~K}$, is constrained by the visibilities with $q>5.3 \mathrm{G} \lambda$. The estimated maximum brightness temperature $T_{\mathrm{b}, \max }=3.1 \times 10^{14} \mathrm{~K}$ is calculated from the data on the same baselines.

\subsection{Brightness temperature}

Table 2 also lists the observed (i.e., not corrected for redshift or Doppler boosting) brightness temperatures, estimated from the model-fitted circular Gaussian components as $T_{\mathrm{b}}=1.36 \times 10^{9} S \lambda^{2} / \theta^{2} \mathrm{~K}$, where $S$ (in Jansky) is the total flux density, $\theta$ (mas) the size, and $\lambda$ (cm) the observing wavelength (e.g., Kovalev et al. 2005). Model-fitted data of the RadioAstron visibilities yield a brightness temperature of $(1.56 \pm 0.26) \times 10^{12} \mathrm{~K}$ for the component upstream the core, "Up", while for the unresolved core component, "Core", we obtain a lower limit of $T_{\mathrm{b}}>2.0 \times 10^{13} \mathrm{~K}$.

The observed brightness temperature in jets is mostly affected by the transverse dimension of the flow and it may differ systematically from estimates obtained on the basis of representing the jet structure with Gaussian components (Lobanov 2015). In this case, constraints on the jet brightness temperature can also be found directly from visibility amplitudes and their errors, providing the minimum brightness temperature, $T_{\mathrm{b}, \min }$, and an estimate of the formal maximum brightness temperature, $T_{\mathrm{b}, \max }$ that can be obtained under condition that the structural detail sampled by the given visibility is resolved. For further details we refer the reader to Lobanov (2015).

These two estimates are compared in Fig. 6 with the brightness temperatures calculated from the Gaussian components described in Table 2. One can see that the observed brightness temperature of the most compact structures in BL Lac, constrained by baselines longer than $5.3 \mathrm{G} \lambda$, must indeed exceed $2 \times 10^{13} \mathrm{~K}$ and can reach as high as $\sim 3 \times 10^{14} \mathrm{~K}$. As follows from Fig. 1 these visibilities correspond to the structural scales of $30-40 \mu \mathrm{as}$ oriented along position angles of $25^{\circ}-30^{\circ}$. These values are indeed close to the width of the inner jet and the normal to its direction.

The observed, $T_{\mathrm{b}, \text { obs }}$, and intrinsic, $T_{\mathrm{b} \text {,int }}$, brightness temperatures are related by

$$
T_{\mathrm{b}, \mathrm{obs}}=\delta(1+z)^{-1} T_{\mathrm{b}, \mathrm{int}}
$$

where $\delta=\left(1-\beta^{2}\right)^{1 / 2}(1-\beta \cos \phi)^{-1}$ is the Doppler factor, $\beta$ is the jet bulk velocity in units of the speed of light, $\phi$ is the jet viewing angle, and $z$ is the redshift of the source. Variability arguments (Jorstad et al. 2005; Hovatta et al. 2009) and kinematical analyses (Cohen et al. 2015) yield a remarkably consistent value of $\delta=7.2$, from which we estimate a lower limit of the intrinsic brightness temperature in the core component of our RadioAstron observations of $T_{\mathrm{b}, \text { int }}>2.9 \times 10^{12} \mathrm{~K}$.

It is commonly considered that inverse Compton losses limit the intrinsic brightness temperature for incoherent synchrotron sources, such as AGN, to about $10^{12} \mathrm{~K}$ 


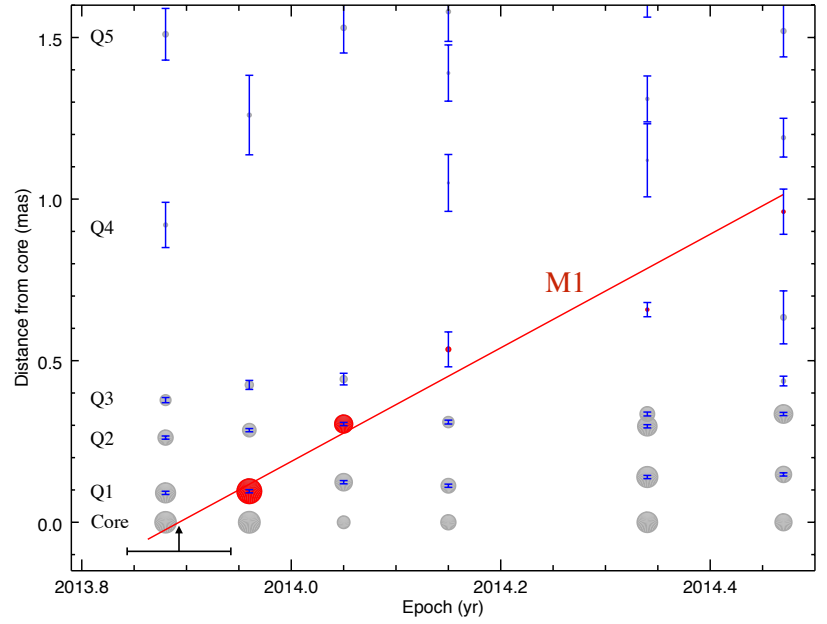

FIG. 7.- VLBA-BU-BLAZAR model fits covering November 2013 through June 2014. Symbol size is proportional to the square root of component's flux density. Marked in red is a new superluminal component, M1, with a proper motion of $7.9 \pm 0.3 c$, ejected from the core in $2013.89 \pm 0.05$ (marked with an arrow and its respective error bar).

(Kellermann \& Pauliny-Toth 1969). In case of a strong flare, the "Compton catastrophe" is calculated to take about one day to drive the brightness temperature below $10^{12} \mathrm{~K}$. Moreover, Readhead (1994) has argued that for sources near equipartition of energy between the magnetic field and radiating particles a more accurate upper value for the intrinsic brightness temperature is about $10^{11} \mathrm{~K}$ (see also Lähteenmäki et al. 1999: Hovatta et al. 2009), which is often called the equipartition brightness temperature.

Our estimated lower limit for the intrinsic brightness temperature of the core in the RadioAstron image of $T_{\mathrm{b} \text {,int }}>2.9 \times 10^{12} \mathrm{~K}$ is therefore more than an order of magnitude larger than the equipartition brightness temperature limit established by Readhead (1994), and at least several times larger than the limit established by inverse Compton cooling. This suggests that the jet in BL Lac is not in equipartition, as may be expected in case the source is flaring during the ejection of component M1 detected at $43 \mathrm{GHz}$, and rises the possibility that we are significantly underestimating its Doppler factor.

We also note that if our estimate of the maximum brightness temperature is closer to actual values, it would imply $T_{\mathrm{b} \text {,int }} \sim 5 \times 10^{13} \mathrm{~K}$. This is difficult to reconcile with current incoherent synchrotron emission models from relativistic electrons, requiring alternative models such as emission from relativistic protons (Jukes 1967), or coherent emission (Benford \& Tzach 2000) - see also Kellermann (2002) and references therein.

\section{POLARIZATION AND FARADAY ROTATION ANALYSIS}

Our polarimetric observations with the ground arrays at 15 and $43 \mathrm{GHz}$ and the space VLBI RadioAstron observations at $22 \mathrm{GHz}$ can be combined to obtain a rotation measure (RM) image of BL Lac. Data at 43 and $22 \mathrm{GHz}$ were first tapered and convolved with a common restoring beam to match the $15 \mathrm{GHz}$ resolution.

Combination of the images at all three frequencies requires also proper registering. Due to the difficulties in finding compact, optically thin components that could be matched across images, we have used for the image

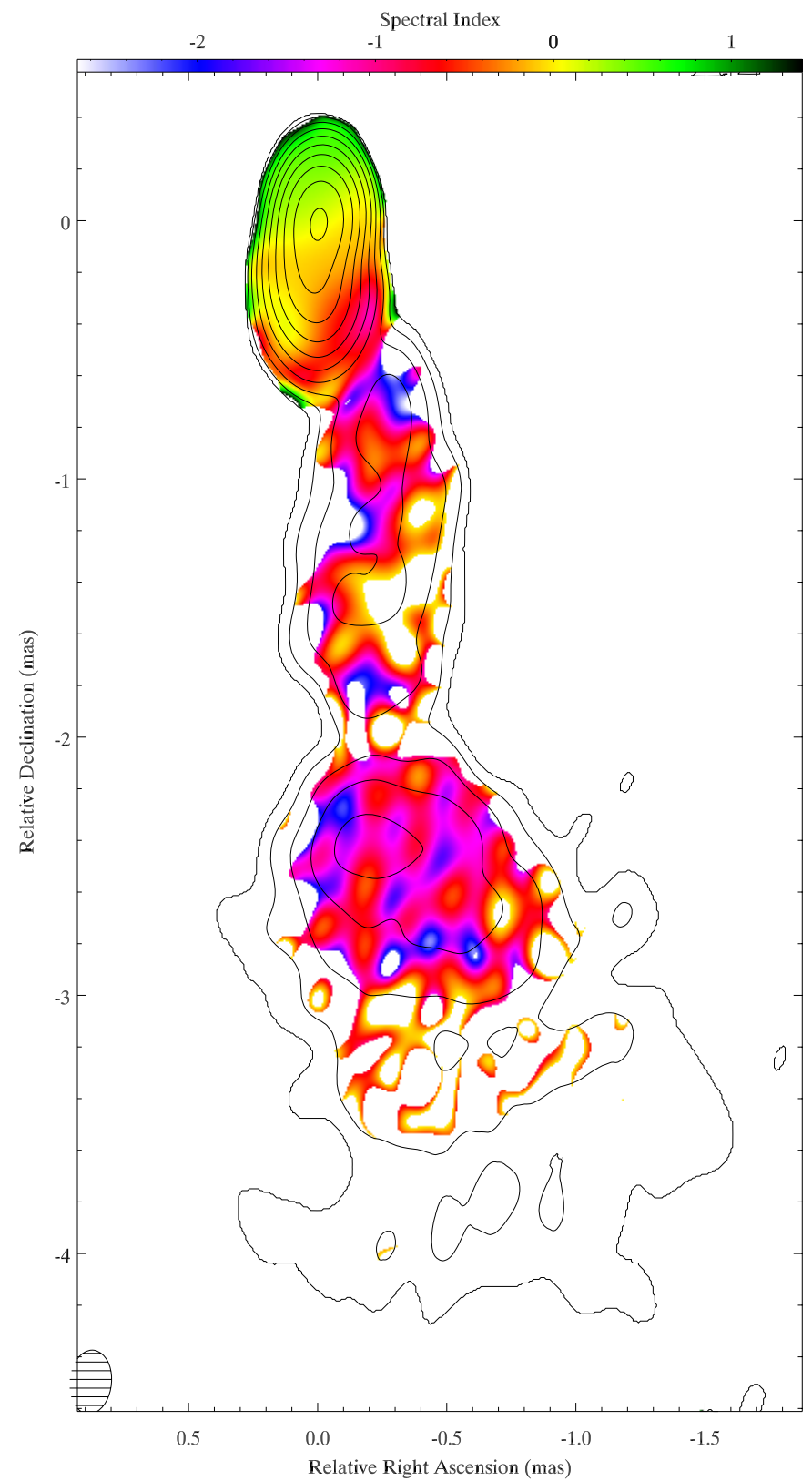

FIG. 8.- Spectral index image between the $22 \mathrm{GHz}$ RadioAstron and $43 \mathrm{GHz}$ ground-array total intensity images. Contours show the total intensity image from the RadioAstron observations convolved with the $43 \mathrm{GHz}$ beam.

alignment a method based on a cross-correlation analysis of the total intensity images (Walker et al. 2000: Croke \& Gabuzda 2008; Hovatta et al. 2012). Only optically thin regions have been considered in the cross-correlation analysis to avoid the shifts in the core position due to opacity (e.g., Lobanov 1998, Kovalev et al. 2008) that could influence our results. We obtain a shift to the south, in the direction of the jet, of 0.021 mas and 0.063 mas for the alignment of the 22 and $15 \mathrm{GHz}$ images, respectively, with respect to the one at $43 \mathrm{GHz}$.

Pixels in the images for which polarization was not detected at all three frequencies simultaneously were blanked. The rotation measure (RM) map is computed by performing a $\lambda^{2}$ fit to the wavelength dependence of the EVPAs at each pixel, blanking pixels with a poor fit 


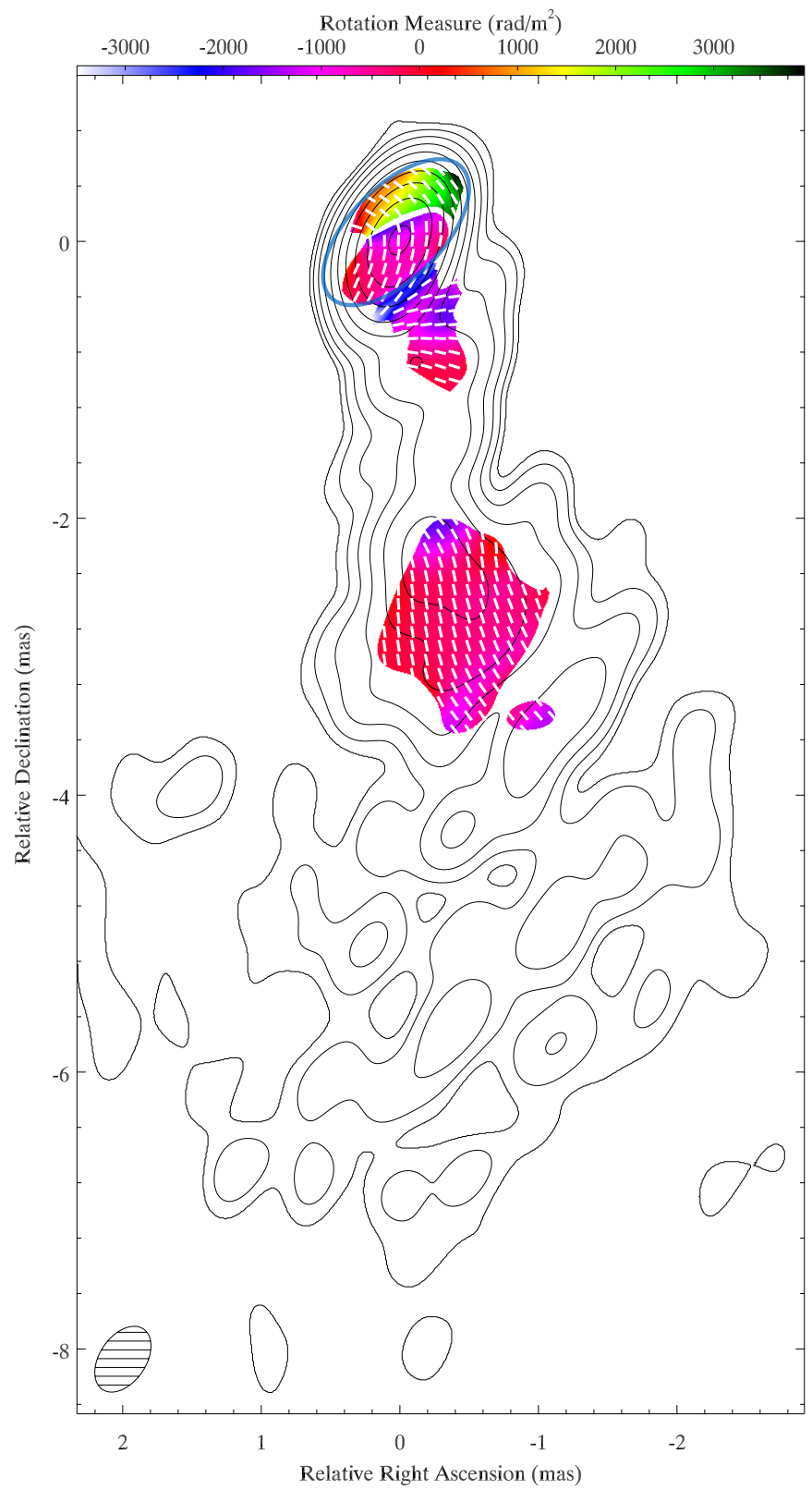

FIG. 9.- Rotation measure map combining the ground-array images at 15 and $43 \mathrm{GHz}$ with the RadioAstron image at $22 \mathrm{GHz}$. Contours show the $15 \mathrm{GHz}$ image, colors indicate the rotation measure, and bars plot the Faraday-corrected EVPAs. The light blue ellipse delimits the core region within which the $2 \mathrm{D}$ histogram images (see Fig. 10 have been computed.

based on a $\chi^{2}$ criterion. Due to the $n \pi$ ambiguity in the EVPAs, we have developed an IDL routine that searches for possible $n \pi$ rotations, finding that no wraps higher than $\pm \pi$ were required to fit the data.

When performing the RM analysis of the core area, it is also important to pay special attention to possible $\pi / 2$ rotations due to opacity (e.g., Gómez et al. 1994 Gabuzda \& Gómez 2001, Porth et al. 2011). We have checked for these by first computing the spectral index maps between each pair of frequencies. Figure 8 shows the spectral index map between the $22 \mathrm{GHz}$ RadioAstron and $43 \mathrm{GHz}$ VLBA images. This reveals an optically thick region at $22 \mathrm{GHz}$ (and therefore also at $15 \mathrm{GHz}$ ) at the upstream end of the jet, near the core. This optically
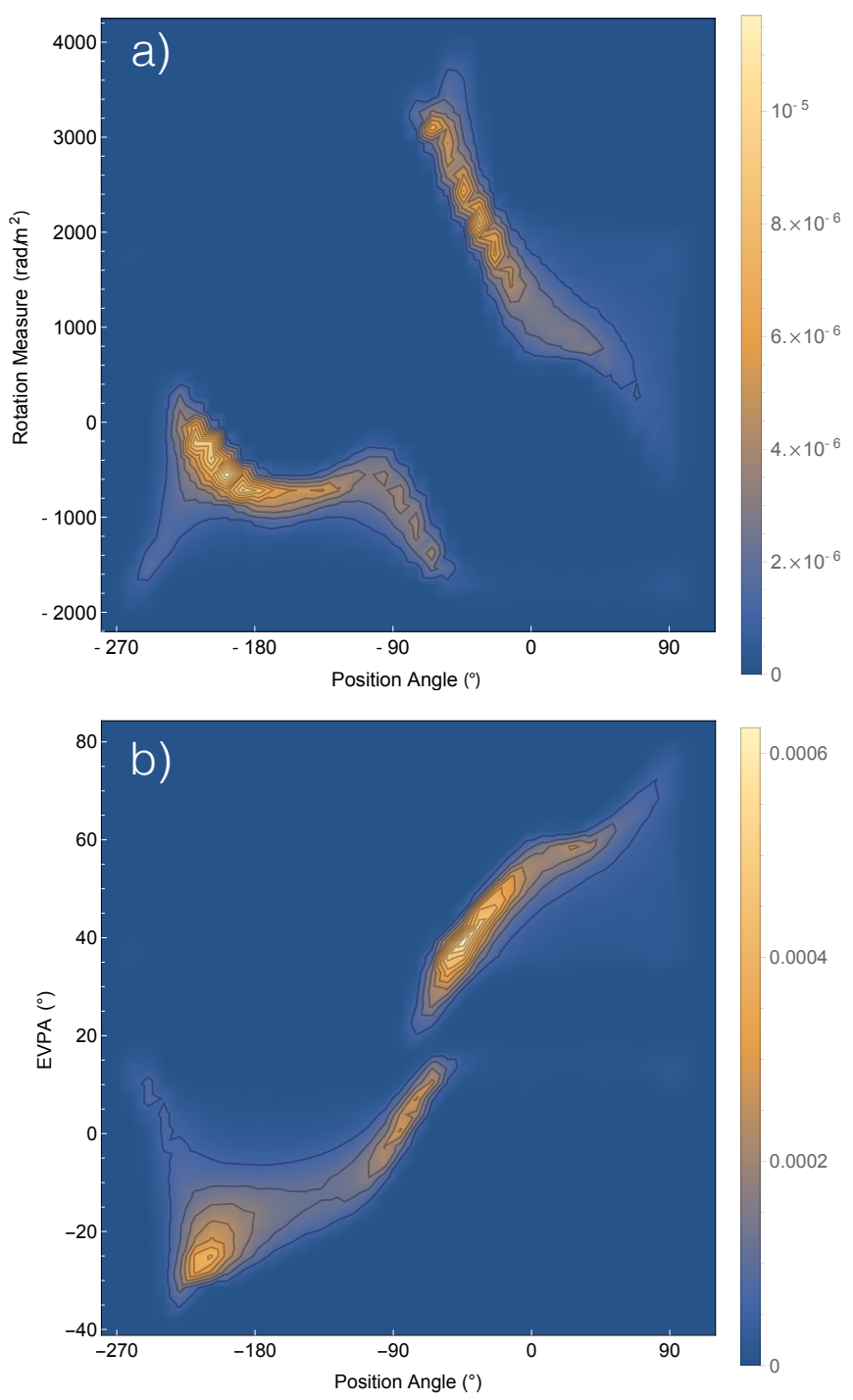

FIG. 10.- Probability density functions of the two-dimensional histograms of the rotation measure ( $a$, top) and Faraday corrected EVPA $(b$, bottom $)$ for the core area indicated by the blue ellipse in Fig. 9

thick region is accounted for when computing the RM map by rotating the EVPAs at 22 and $15 \mathrm{GHz}$ by $\pi / 2$. The resulting rotation measure map is shown in Fig. 9

\subsection{Evidence for a helical magnetic field}

The rotation measure and RM-corrected EVPAs $\left(\chi_{0}\right)$ in the core area (delimited by the blue ellipse in Fig. 9 ) exhibit a clear point symmetry around its centroid. To better analyze this structure Fig. 10 displays the probability distribution function of the two-dimensional histogram for the RM and $\chi_{0}$ as a function of the position angle of the pixels with respect to the centroid of the core, measured counterclockwise from north.

By inspecting Figs. 9 and 10 a we note a gradient in RM with position angle from the centroid of the core, with positive RM values in the area upstream of the centroid, and negative downstream, in the direction of the jet. The largest RM values, of the order of $3000 \mathrm{rad} \mathrm{m}^{-2}$, are found in the area northeast of the centroid (with a position angle of $\sim-45^{\circ}$ ); smaller values are obtained as the position angle increases, reaching values of $\sim 1000$ 

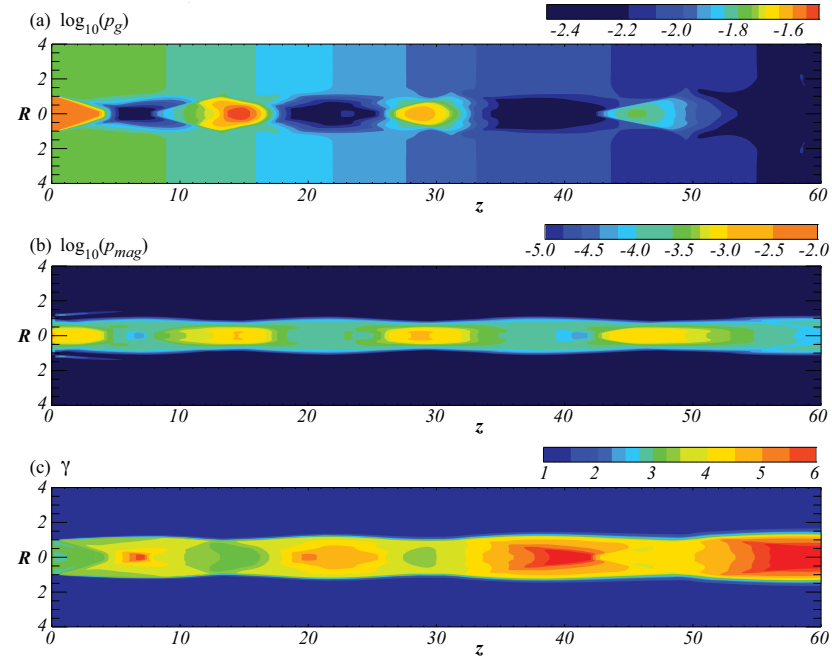

(d)
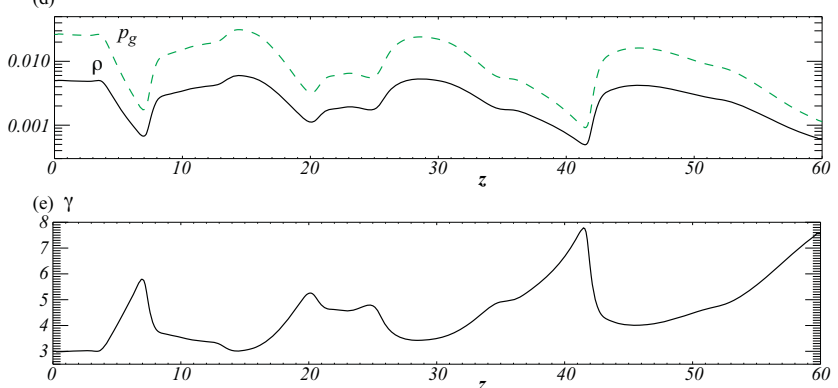

FIG. 11.- Relativistic magnetohydrodynamic simulations for an over-pressured jet propagating from left to right with a helical magnetic field at $t_{\mathrm{s}}=600$, where $t_{\mathrm{s}}$ is in units of $R_{\mathrm{j}} / c$. Upper panels: 2D plots of: $(a)$ the gas pressure density, $(b)$ the magnetic pressure $\left(p_{\text {mag }}=B^{2} / 2\right)$, and $(c)$ the Lorentz factor. Lower panels: $1 \mathrm{D}$ profiles along the jet axis $(R=0)$ of: $(d)$ the rest-mass density (solid) and the gas pressure (green dashed), and $(e)$ the Lorentz factor.

rad $\mathrm{m}^{-2}$ north-west of the centroid. Downstream of the centroid the RM continues this trend, reaching values of $\sim-900 \mathrm{rad} \mathrm{m}^{-2}$ in the direction of the jet.

Similarly, Figs. 9 and 10p show a progressive rotation in $\chi_{0}$ with position angle from the centroid of the core. Counterclockwise from east, $\chi_{0}$ rotate continuously from $\sim-25^{\circ}$ to $\sim 60^{\circ}$. On top of this, the two-dimensional histogram shows a concentration of $\chi_{0}$ values between approximately $30^{\circ}$ and $50^{\circ}$ in the area upstream of the centroid, while downstream these concentrate in values at $\sim-25^{\circ}$ and $0^{\circ}$.

A similar dependence of the RM and $\chi_{0}$ with polar angle was found by Zamaninasab et al. (2013) in 3C 454.3, interpreted by these authors as the result of a helical magnetic field. Indeed, gradients in RM across the jet width are expected to arise in the case of helical magnetic fields (Laing 1981), as previously reported in a number of sources (e.g., Asada et al. 2002, O'Sullivan \& Gabuzda 2009, Hovatta et al. 2012).

Relativistic magnetohydrodynamic (RMHD) simulations have been used to study the rotation measure and polarization distribution based on self-consistent models for jet formation and propagation in the presence of large scale helical magnetic fields (Broderick \& McKinney 2010, Porth et al. 2011). These simulations reproduce the expected gradients in RM across the jet width due to the toroidal component of the helical magnetic field, and provide also detailed insights regarding the po-

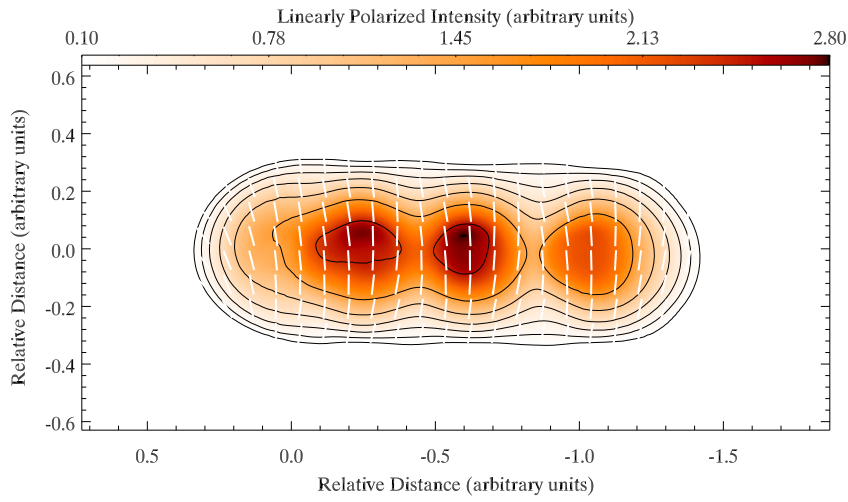

FIG. 12.- Simulated total intensity (contours, in logarithmic scale), linearly polarized intensity (colors), and EVPAs (bars) obtained by computing the (optically thin) radio continuum synchrotron emission at a viewing angle of $10^{\circ}$ using the RMHD model shown in Fig. 11] as input.

larization structure throughout the jet, which depends strongly on the helical magnetic field pitch angle, jet viewing angle, Lorentz factor, and opacity.

As discussed in Zamaninasab et al. (2013), Broderick \& McKinney (2010), and Porth et al. (2011), a largescale helical magnetic field would lead to similar point symmetric structures around the centroid of the core of both RM and $\chi_{0}$ as found in Figs. 9 and 10 . This suggests that the core region in BL Lac is threaded by a large-scale helical magnetic field. A more detailed comparison between our observations and specific RMHD simulations using the estimated parameters for BL Lac is underway and will be published elsewhere.

\subsection{Pattern of recollimation shocks}

The location of the stationary feature $\sim 0.26$ mas from the core (see Sect. 3.1) marks a clear transition in the RM and polarization vectors between the core area and the remainder of the jet in BL Lac. Figure 9 shows a localized region of enhanced RM, reaching values of the order of $-2200 \pm 300 \mathrm{rad} \mathrm{m}^{-2}$, and a Faraday rotation corrected EVPA of $-40 \pm 8^{\circ} . \chi_{0}$ therefore becomes perpendicular to the local jet direction, suggesting a dominant component of the magnetic field that is aligned with the jet. Downstream of this location, $\chi_{0}$ rotates so that the magnetic field remains predominantly aligned with the local jet direction up to a distance of $\sim 1$ mas from the core.

Further downstream polarization is detected again in a region at a distance of $\sim 3$ mas from the core, corresponding to the location of components K6 and K7 (see Fig. 5). This area has a mean RM and $\chi_{0}$ of $-320 \pm 140 \mathrm{rad} \mathrm{m}^{-2}$ and $14 \pm 6^{\circ}$, respectively. The polarization vectors are approximately parallel to the jet in this area, thereby suggesting that the magnetic field is predominantly perpendicular to the jet. This would be in agreement with a helical magnetic field in which the toroidal component dominates over the poloidal one, although other scenarios, like a plane perpendicular shock, cannot be ruled out.

Considering that the polarization properties in the core area and components $\mathrm{K} 6$ and $\mathrm{K} 7$ suggest that the jet in BL Lac is threaded by a helical magnetic field, the different polarization structure associated with the stationary feature at $\sim 0.26$ mas from the core suggests that it may correspond to a rather particular jet feature. This would 
be in agreement with claims by Cohen et al. (2015), and references therein, in which these authors conclude that this stationary feature corresponds to a recollimation shock, downstream of which new superluminal components appear due to the propagation of Alfvén waves triggered by the swing in its position, in a similar way as exciting a wave on a whip by shaking the handle.

Previous multiwavelength observations of BL Lac suggest that the core may also correspond to a recollimation shock (Marscher et al. 2008). In that case we can hypothesize that the upstream component found in our RadioAstron observation (see Fig. 5 and Table 2 ) is located at the jet apex, so that the distance at which the first recollimation shock takes place, associated with the core, would be $\sim 40 \mu$ as. If so, the jet in BL Lac would contain a set of three recollimation shocks, spaced at progressively larger distances of approximately 40, 100, and $250 \mu$ as.

To investigate such a pattern of recollimation shocks as possibly observed in BL Lac, we have performed twodimensional special relativistic magnetohydrodynamic simulations in cylindrical geometry using the RAISHIN code (Mizuno et al. 2006, 2011). The initial set-up follows Gómez et al. (1997) and Mizuno et al. (2015), in which a preexisting over-pressured How is established across the simulation domain. We choose a rest-mass density ratio between the jet and ambient medium of $\eta=\rho_{\mathrm{j}} / \rho_{\mathrm{a}}=5 \times 10^{-3}$ with $\rho_{\mathrm{a}}=1.0 \rho_{0}$, initial jet Lorentz factor $\gamma_{\mathrm{j}}=3$, and local Mach number $M_{\mathrm{s}}=1.69$. The gas pressure in the ambient medium decreases with axial distance from the jet base following $p_{\mathrm{g}, \mathrm{a}}(z)=p_{\mathrm{g}, 0} /[1+$ $\left.\left(z / z_{\mathrm{c}}\right)^{n}\right]^{m / n}$, where $z_{\mathrm{c}}=60 R_{\mathrm{j}}$ is the gas pressure scale height in the axial direction, $R_{\mathrm{j}}$ is the jet radius, $n=1.5$, $m=2.3$, and $p_{\mathrm{g} .0}$ is in units of $\rho_{0} c^{2}$ (e.g., Gómez et al. 1995, 1997; Mimica et al. 2009). We assume that the jet is initially uniformly over-pressured with $p_{\mathrm{g}, \mathrm{j}}=1.5 p_{\mathrm{g}, \mathrm{a}}$. We consider a force-free helical magnetic field with a weak magnetization $B_{0}=0.05$ (in units of $\sqrt{4 \pi \rho_{0} c^{2}}$ ) and constant magnetic pitch $P_{0}=R B_{z} / R_{\mathrm{j}} B_{\phi}=1 / 2$, where $B_{z}$ and $B_{\phi}$ are the poloidal and toroidal magnetic field components, so that smaller $P_{0}$ refers to increased magnetic helicity.

Overexpansions and overcontractions caused by inertial overshooting past equilibrium of the jet lead to the formation of a pattern of standing oblique recollimation shocks and rarefactions, whose strength and spacing are governed by the external pressure gradient (see Fig. 11). The jet is accelerated and conically expanded slightly by the gas pressure gradient force, reaching a maximum jet Lorentz factor of $\sim 8$. The jet radius expands from the initial $R_{\mathrm{j}}=1$ at the jet base to $R_{\mathrm{j}}=1.5$ at $z=60 R_{\mathrm{j}}$, yielding an opening angle of $\theta_{\mathrm{j}}=\arctan \left(0.5 R_{\mathrm{j}} / 60 R_{\mathrm{j}}\right) \sim 0.48^{\circ}$. The recollimation shocks are located at progressively larger axial distances of $\sim 7 R_{\mathrm{j}}, \sim 20 R_{\mathrm{j}}$, and $\sim 42 R_{\mathrm{j}}$. The relative distances between the second and third shocks with respect to the first one are $\sim 2.9$ and $\sim 6$, which roughly match those observed in BL Lac.

The output from the RMHD simulation is used as input to compute the radio continuum synchrotron emission map shown in Fig. 12. Following Gómez et al. (1995), the internal energy is distributed among the non-thermal electrons using a power law $N(E) d E=$
$N_{0} E^{-p} d E$, with $p=2.4$. The emission is computed for a viewing angle of $10^{\circ}$ and at an optically thin observing frequency, integrating the synchrotron transfer equations along the line of sight (e.g., Gómez et al.1997). The pattern of recollimation shocks seen in Fig. 11 leads to a set of knots in the total and linearly polarized intensity. The EVPAs in the knots are perpendicular to the jet direction, in agreement with the observations of component $\mathrm{K} 2$ in BL Lac. We note however that our RMHD simulations consider an already formed and collimated jet, and therefore do not provide an accurate account of the jet formation region, close to where the other two innermost recollimation shocks are located.

Finally, although our simulations are in general agreement with the pattern of recollimation shocks observed in BL Lac, further numerical simulations, in progress, are required to better constrain the jet parameters of the model, such as the Lorentz factor, Mach number, gradient in external pressure, magnetic field helicity/strength, and viewing angle.

\section{SUMMARY}

RadioAstron provides the first true full-polarization capabilities for space VLBI observations on baselines longer than the Earth's diameter, opening the possibility to achieve unprecedentedly high angular resolution in astronomical imaging. In this paper we present the first polarimetric space VLBI observations at $22 \mathrm{GHz}$, obtained as part of our RadioAstron KSP designed to probe the innermost regions of AGN and their magnetic fields in the vicinity of the central black hole.

The jet of BL Lac was observed in 2013 November 10 at $22 \mathrm{GHz}$ with RadioAstron including a ground array of 15 radio telescopes. The instrumental polarization of the space radio telescope was found to remain within $9 \%$ ( $5 \%$ for LCP), demonstrating its polarization capabilities for RadioAstron observations at its highest observing frequency of $22 \mathrm{GHz}$.

The phasing of a group of ground-based antennas allowed to obtain reliable ground-space fringe detections up to projected baseline distances of 7.9 Earth's diameters in length. Polarization images of BL Lac are obtained with a maximum angular resolution of $21 \mu \mathrm{as}$, the highest achieved to date.

Analysis of the $43 \mathrm{GHz}$ data from the VLBA-BUBLAZAR monitoring program, covering from November 2013 to June 2014, reveals a new component ejected near the epoch of our RadioAstron observations, confirmed also by flares in the optical and $\gamma$-ray light curves. This new component appears in the RadioAstron image as a knot $41 \pm 3 \mu$ as upstream of the radio core, in agreement with previous detections of upstream emission in BL Lac (Marscher et al. 2008). The radio core would then correspond to a recollimation shock at $\sim 40 \mu$ as from the jet apex, part of a pattern that also includes two other recollimation shocks at approximately 100 and $250 \mu$ as. Our relativistic magnetohydrodynamic simulations show that such a pattern of recollimation shocks, spaced at progressively larger distance, is expected when the jet propagates through an ambient medium with a decreasing pressure gradient.

Polarization is detected in two components within the innermost 0.5 mas of the core, and in some knots $\sim 3$ mas downstream. We have combined the RadioAstron 
$22 \mathrm{GHz}$ image with ground-based observations at 43 and $15 \mathrm{GHz}$ to compute a rotation measure map. Analysis of the core area shows a gradient in rotation measure and Faraday corrected EVPA that depends on the position angle with respect to the core, in similar way as found in the jet of 3C 454.3 (Zamaninasab et al. 2013), and in agreement with numerical RMHD simulations of jets threaded by a helical magnetic field (Broderick \& McKinney 2010, Porth et al. 2011).

The stationary feature $\sim 250 \mu$ as from the core contains an enhanced rotation measure and marks a temporary transition in polarization vectors, in which the magnetic field becomes predominantly aligned with the local direction of the jet, in agreement with our RMHD simulations.

We obtain a lower limit for the observed brightness temperature of the unresolved core in our RadioAstron image of $2 \times 10^{13} \mathrm{~K}$. Using previous estimates of the Doppler factor of $\delta=7.2$ we calculate an intrinsic brightness temperature in excess of $3 \times 10^{12} \mathrm{~K}$, implying that the jet in BL Lac is not in equipartition of energy between the magnetic field and emitting particles, and suggesting also that its Doppler factor is significantly underestimated.

RadioAstron polarimetric space VLBI observations provide a unique tool to study the innermost regions of AGN jets and their magnetic fields with unprecedented high angular resolutions. This will be investigated in a series of papers, results from our RadioAstron Key Science Program, which studies a sample of powerful, highly polarized, and $\gamma$-ray emitting blazars.

We thank José M. Martí, Timothy V. Cawthorne, Oliver Porth, and Carolina Casadio for valuable comments that improved our manuscript. This research has been supported by the Spanish Ministry of Economy and Competitiveness grant AYA2013-40825-P, by the Russian Foundation for Basic Research (projects 1302-12103, 14-02-31789, and 15-02-00949), and St. Petersburg University research grant 6.38.335.2015. The research at Boston University (BU) was funded in part by NASA Fermi Guest Investigator grant NNX14AQ58G. Y. M. acknowledges support from the ERC Synergy Grant "BlackHoleCam - Imaging the Event Horizon of Black Holes" (Grant 610058). Part of this work was supported by the COST Action MP1104 "Polarization as a tool to study the Solar System and beyond". The RadioAstron project is led by the Astro Space Center of the Lebedev Physical Institute of the Russian Academy of Sciences and the Lavochkin Scientific and Production Association under a contract with the Russian Federal Space Agency, in collaboration with partner organizations in Russia and other countries. This research is based on observations correlated at the Bonn Correlator, jointly operated by the Max-Planck-Institut für Radioastronomie (MPIfR), and the Federal Agency for Cartography and Geodesy (BKG). The European VLBI Network is a joint facility of of independent European, African, Asian, and North American radio astronomy institutes. Scientific results from data presented in this publication are derived from the EVN project code GA030B. This research is partly based on observations with the $100 \mathrm{~m}$ telescope of the MPIfR at Effelsberg. The VLBA is an instrument of the National Radio Astronomy Observatory, a facility of the National Science Foundation operated under cooperative agreement by Associated Universities. The relativistic magnetohydrodynamic simulations were performed on Pleiades at NASA and on LOEWE at the Goethe University Frankfurt.

Facilities: RadioAstron Space Radio Telescope (Spektr-R), EVN, VLBA

\section{REFERENCES}

Asada, K., Inoue, M., Uchida, Y., et al. 2002, PASJ, 54, L39

Benford, G., \& Tzach, D. 2000, MNRAS, 317, 497

Blandford, R. D., \& Payne, D. G. 1982, MNRAS, 199, 883

Blandford, R. D., \& Znajek, R. L. 1977, MNRAS, 179, 433

Broderick, A. E., \& McKinney, J. C. 2010, ApJ, 725, 750

Bruni, G., Anderson, J., Alef, W., Lobanov, A. P., \& Zensus,

A. J. 2014, in Proceedings of the 12th European VLBI Network Symposium, Proceedings of Science, 119

Casadio, C., Gómez, J. L., Jorstad, S. G., et al. 2015, ApJ, 813, 51

Cohen, M. H., Meier, D. L., Arshakian, T. G., et al. 2014, ApJ,

787,151

-. 2015, ApJ, 803, 3

Croke, S. M., \& Gabuzda, D. C. 2008, MNRAS, 386, 619

Ford, H. A., Anderson, R., Belousov, K., et al. 2014, in

Proceedings of the SPIE, ed. L. M. Stepp, R. Gilmozzi, \& H. J. Hall, National Radio Astronomy Observatory (United States) (SPIE), 91450B

Gabuzda, D. C., \& Gómez, J. L. 2001, MNRAS, 320, L49

Gómez, J. L., Alberdi, A., Marcaide, J. M., Marscher, A. P., \& Travis, J. P. 1994, A\&A, 292, 33

Gómez, J. L., \& Marscher, A. P. 2000, ApJ, 530, 245

Gómez, J. L., Martí, J. M., Marscher, A. P., Ibáñez, J. M., \& Alberdi, A. 1997, ApJ, 482, L33

Gómez, J. L., Marti, J. M. A., Marscher, A. P., Ibanez, J. M. A., \& Marcaide, J. M. 1995, ApJ, 449, L19

Hovatta, T., Lister, M. L., Aller, M. F., et al. 2012, AJ, 144, 105

Hovatta, T., Valtaoja, E., Tornikoski, M., \& Lähteenmäki, A. 2009, A\&A, 494, 527

Jorstad, S. G., Marscher, A. P., Lister, M. L., et al. 2005, AJ, 130,1418
Jorstad, S. G., Marscher, A. P., Stevens, J. A., et al. 2007, AJ, 134,799

Jukes, J. D. 1967, Nature, 216, 461

Kardashev, N. S., Khartov, V. V., Abramov, V. V., et al. 2013, Astronomy Reports, 57, 153

Kellermann, K. I. 2002, PASA, 19, 77

Kellermann, K. I., \& Pauliny-Toth, I. I. K. 1969, ApJ, 155, L71

Kogan, L. 1996, VLBA Scientific Memo, \# 13

Kovalev, Y. A., Vasil'kov, V. I., Popov, M. V., et al. 2014, Cosmic Res, 52, 393

Kovalev, Y. Y., Lobanov, A. P., Pushkarev, A. B., \& Zensus, J. A. 2008, A\&A, 483, 759

Kovalev, Y. Y., Kellermann, K. I., Lister, M. L., et al. 2005, AJ, 130,2473

Lähteenmäki, A., Valtaoja, E., \& Wiik, K. 1999, ApJ, 511, 112

Laing, R. A. 1981, ApJ, 248, 87

Leppanen, K. J., Zensus, J. A., \& Diamond, P. J. 1995, AJ, 110, 2479

Levy, G. S., Linfield, R. P., Ulvestad, J. S., et al. 1986, Science, 234,187

Lind, K. R., Payne, D. G., Meier, D. L., \& Blandford, R. D. 1989, Astrophysical Journal, 344, 89

Lister, M. L., Aller, M. F., Aller, H. D., et al. 2013, AJ, 146, 120

Lobanov, A. 2015, A\&A, 574, A84

Lobanov, A. P. 1998, A\&A, 330, 79

Lobanov, A. P., \& Zensus, J. A. 2001, Science, 294, 128

Lobanov, A. P., Gómez, J. L., Bruni, G., et al. 2015, A\&A, 583, A100

Marscher, A. P., Jorstad, S. G., Gómez, J. L., et al. 2002, Nature, 417,625 
Marscher, A. P., Jorstad, S. G., D'Arcangelo, F. D., et al. 2008, Nature, 452, 966

McKinney, J. C., \& Blandford, R. D. 2009, MNRAS, 394, L126

Meier, D. L. 2013, In The Innermost Regions of Relativistic Jets and Their Magnetic Fields, EPJ Web of Conferences, 61, 01001

Mimica, P., Aloy, M. A., Agudo, I., et al. 2009, ApJ, 696, 1142

Mizuno, Y., Gómez, J. L., Nishikawa, K.-I., et al. 2015, ApJ, 809, 38

Mizuno, Y., Hardee, P. E., \& Nishikawa, K.-I. 2011, ApJ, 734, 19

Mizuno, Y., Nishikawa, K.-I., Koide, S., Hardee, P., \& Fishman, G. J. 2006, arXiv:astro-ph/0609004

Mutel, R. L., \& Denn, G. R. 2005, ApJ, 623, 79

O'Sullivan, S. P., \& Gabuzda, D. C. 2009, MNRAS, 393, 429

Pashchenko, I. N., Kovalev, Y. Y., \& Voitsik, P. A. 2015, Cosmic Res, 53, 199

Planck Collaboration, Ade, P. A. R., Aghanim, N., et al. 2014, A\&A, 571, A16

Porth, O., Fendt, C., Meliani, Z., \& Vaidya, B. 2011, ApJ, 737, 42

Pushkarev, A. B., Hovatta, T., Kovalev, Y. Y., et al. 2012, A\&A, 545,113
Pushkarev, A. B., \& Kovalev, Y. Y. 2015, MNRAS, 452, 4274

Readhead, A. C. S. 1994, ApJ, 426, 51

Shepherd, M. C. 1997, in ASP Conf. Ser. 125, Astronomical Data Analysis Software and Systems VI, ed. G. Hunt \& H. E. Payne (San Francisco: ASP), 77

Sokolovsky, K. V., Kovalev, Y. Y., Pushkarev, A. B., \& Lobanov, A. P. 2011, A\&A, 532, 38

Stirling, A. M., Cawthorne, T. V., Stevens, J. A., et al. 2003, MNRAS, 341, 405

Walker, R. C., Dhawan, V., Romney, J. D., Kellermann, K. I., \& Vermeulen, R. C. 2000, ApJ, 530, 233

Woo, J.-H., \& Urry, C. M. 2002, ApJ, 579, 530

Zamaninasab, M., Clausen-Brown, E., Savolainen, T., \&

Tchekhovskoy, A. 2014, Nature, 510, 126

Zamaninasab, M., Savolainen, T., Clausen-Brown, E., et al. 2013, MNRAS, 436, 3341

Zavala, R. T., \& Taylor, G. B. 2003, ApJ, 589, 126 
TABLE 3

Gaussian model fits for the VLBA-BU-BLAZAR 43 GHz DATA

\begin{tabular}{|c|c|c|c|c|}
\hline $\begin{array}{l}\text { Epoch } \\
\text { (year) }\end{array}$ & $\begin{array}{l}\text { Flux } \\
(\mathrm{Jy})\end{array}$ & $\begin{array}{c}\text { Distance } \\
\text { (mas) }\end{array}$ & $\begin{array}{l}\text { PA } \\
\left({ }^{\circ}\right)\end{array}$ & $\begin{array}{l}\text { Size } \\
(\mathrm{mas})\end{array}$ \\
\hline \multirow[t]{8}{*}{2013.96} & $1.620 \pm 0.087$ & & & $0.044 \pm 0.005$ \\
\hline & $2.100 \pm 0.111$ & $0.096 \pm 0.005$ & $-174 \pm 5$ & $0.062 \pm 0.006$ \\
\hline & $0.620 \pm 0.039$ & $0.285 \pm 0.005$ & $178 \pm 1$ & $0.087 \pm 0.007$ \\
\hline & $0.227 \pm 0.021$ & $0.425 \pm 0.014$ & $179 \pm 2$ & $0.132 \pm 0.005$ \\
\hline & $0.049 \pm 0.010$ & $1.260 \pm 0.123$ & $-167 \pm 6$ & $0.370 \pm 0.019$ \\
\hline & $0.084 \pm 0.016$ & $1.660 \pm 0.057$ & $-170 \pm 2$ & $0.256 \pm 0.012$ \\
\hline & $0.129 \pm 0.020$ & $2.590 \pm 0.100$ & $-173 \pm 2$ & $0.507 \pm 0.025$ \\
\hline & $0.097 \pm 0.020$ & $3.790 \pm 0.300$ & $-166 \pm 6$ & $1.390 \pm 0.070$ \\
\hline \multirow[t]{8}{*}{2014.05} & $0.547 \pm 0.028$ & & & $<0.005$ \\
\hline & $1.040 \pm 0.059$ & $0.124 \pm 0.005$ & $-170 \pm 2$ & $0.063 \pm 0.005$ \\
\hline & $1.110 \pm 0.063$ & $0.304 \pm 0.005$ & $-179 \pm 1$ & $0.104 \pm 0.005$ \\
\hline & $0.168 \pm 0.018$ & $0.443 \pm 0.018$ & $178 \pm 2$ & $0.140 \pm 0.007$ \\
\hline & $0.097 \pm 0.017$ & $1.530 \pm 0.078$ & $-169 \pm 3$ & $0.356 \pm 0.018$ \\
\hline & $0.054 \pm 0.011$ & $1.960 \pm 0.100$ & $-168 \pm 3$ & $0.322 \pm 0.016$ \\
\hline & $0.043 \pm 0.009$ & $2.480 \pm 0.084$ & $-176 \pm 2$ & $0.255 \pm 0.012$ \\
\hline & $0.105 \pm 0.019$ & $2.990 \pm 0.150$ & $-172 \pm 3$ & $0.652 \pm 0.033$ \\
\hline \multirow[t]{10}{*}{2014.15} & $0.785 \pm 0.045$ & & & $0.028 \pm 0.005$ \\
\hline & $0.735 \pm 0.043$ & $0.113 \pm 0.005$ & $-166 \pm 3$ & $0.048 \pm 0.005$ \\
\hline & $0.434 \pm 0.030$ & $0.310 \pm 0.006$ & $-176 \pm 1$ & $0.091 \pm 0.005$ \\
\hline & $0.070 \pm 0.015$ & $0.535 \pm 0.054$ & $172 \pm 6$ & $0.224 \pm 0.011$ \\
\hline & $0.010 \pm 0.003$ & $1.050 \pm 0.088$ & $172 \pm 5$ & $0.126 \pm 0.007$ \\
\hline & $0.021 \pm 0.005$ & $1.390 \pm 0.087$ & $-179 \pm 4$ & $0.182 \pm 0.010$ \\
\hline & $0.058 \pm 0.015$ & $1.580 \pm 0.092$ & $-170 \pm 3$ & $0.318 \pm 0.016$ \\
\hline & $0.065 \pm 0.016$ & $1.960 \pm 0.100$ & $-170 \pm 3$ & $0.357 \pm 0.018$ \\
\hline & $0.037 \pm 0.010$ & $2.520 \pm 0.130$ & $-175 \pm 3$ & $0.332 \pm 0.016$ \\
\hline & $0.133 \pm 0.020$ & $3.180 \pm 0.160$ & $-172 \pm 3$ & $0.755 \pm 0.038$ \\
\hline \multirow[t]{11}{*}{2014.34} & $1.450 \pm 0.078$ & & & $0.026 \pm 0.005$ \\
\hline & $1.510 \pm 0.082$ & $0.140 \pm 0.005$ & $-161 \pm 2$ & $0.049 \pm 0.006$ \\
\hline & $1.290 \pm 0.071$ & $0.297 \pm 0.005$ & $-162 \pm 1$ & $0.054 \pm 0.007$ \\
\hline & $0.739 \pm 0.045$ & $0.335 \pm 0.006$ & $-172 \pm 1$ & $0.113 \pm 0.006$ \\
\hline & $0.027 \pm 0.007$ & $0.658 \pm 0.022$ & $176 \pm 1$ & $0.031 \pm 0.005$ \\
\hline & $0.013 \pm 0.003$ & $1.120 \pm 0.113$ & $171 \pm 6$ & $0.175 \pm 0.008$ \\
\hline & $0.030 \pm 0.008$ & $1.310 \pm 0.071$ & $178 \pm 3$ & $0.184 \pm 0.010$ \\
\hline & $0.111 \pm 0.018$ & $1.640 \pm 0.077$ & $-176 \pm 3$ & $0.374 \pm 0.020$ \\
\hline & $0.025 \pm 0.006$ & $2.290 \pm 0.100$ & $-167 \pm 3$ & $0.224 \pm 0.011$ \\
\hline & $0.040 \pm 0.010$ & $2.650 \pm 0.110$ & $-173 \pm 2$ & $0.305 \pm 0.015$ \\
\hline & $0.141 \pm 0.021$ & $3.670 \pm 0.200$ & $-171 \pm 3$ & $0.967 \pm 0.048$ \\
\hline \multirow[t]{11}{*}{2014.47} & $0.991 \pm 0.053$ & & & $<0.005$ \\
\hline & $0.902 \pm 0.052$ & $0.148 \pm 0.005$ & $-165 \pm 2$ & $0.058 \pm 0.005$ \\
\hline & $1.180 \pm 0.066$ & $0.335 \pm 0.005$ & $-164 \pm 1$ & $0.066 \pm 0.005$ \\
\hline & $0.055 \pm 0.011$ & $0.437 \pm 0.015$ & $-156 \pm 1$ & $0.047 \pm 0.006$ \\
\hline & $0.104 \pm 0.017$ & $0.634 \pm 0.082$ & $-177 \pm 7$ & $0.383 \pm 0.019$ \\
\hline & $0.030 \pm 0.007$ & $0.961 \pm 0.070$ & $167 \pm 4$ & $0.176 \pm 0.009$ \\
\hline & $0.049 \pm 0.012$ & $1.190 \pm 0.060$ & $174 \pm 3$ & $0.202 \pm 0.010$ \\
\hline & $0.084 \pm 0.016$ & $1.520 \pm 0.080$ & $-177 \pm 3$ & $0.332 \pm 0.026$ \\
\hline & $0.047 \pm 0.012$ & $1.980 \pm 0.200$ & $-175 \pm 6$ & $0.524 \pm 0.023$ \\
\hline & $0.044 \pm 0.011$ & $2.660 \pm 0.170$ & $-173 \pm 4$ & $0.459 \pm 0.023$ \\
\hline & $0.128 \pm 0.021$ & $3.740 \pm 0.220$ & $-171 \pm 3$ & $0.960 \pm 0.048$ \\
\hline
\end{tabular}

Notes. Tabulated data correspond to flux density, distance and position angle from the core, and size. Errors in the model-fit parameters for each component are estimated based on its brightness temperature following Casadio et al. (2015). 